\title{
Die Rolle der Computerisierung und Digitalisierung für Beschäftigung und Einkommen*
}

\author{
Stephan L. Thomsen ${ }^{\dagger}$ \\ Leibniz Universität Hannover, ZEW M annheim und IZA Bonn
}

November 2018

\begin{abstract}
Zusammenfassung
Die Entwicklung der Computer und die darauf aufbauende Digitalisierung haben technologische Veränderungen ausgelöst, die die Anforderungen an und die Nachfrage nach Arbeit, aber auch das Arbeitsangebot in den letzten Dekaden stetig verändert haben. Wie groß die kausalen Effekte dieser Entwicklung auf die Einkommens- und Vermögensverteilung sind, ist in der ökonomischen Literatur bisher weder eindeutig, noch abschließend geklärt. Schwierigkeiten in der Bestimmung dieser Effekte haben sich zunächst aus dem unvollständigen Verständnis ergeben, wie die Computerisierung und die Digitalisierung die Wertschöpfung beeinflusst haben bzw. welchen Beitrag sie leisten. Dies hat zugleich zu der grundsätzlichen Frage geführt, inwiefern der technologische Wandel durch die Computerisierung und Digitalisierung mit traditionellen ökonomischen M odellen, die Arbeit, Kapital und technischen Fortschritt in der Regel als trennbare Faktoren unterscheiden und hierbei feste Relationen bzw. proportionale Effekte unterstellen, erfasst werden kann.

Das Verständnis der Bedeutung und Begründung technischen Fortschritts in seinen Wirkungen für Wirtschaft und Gesellschaft ist dabei bereits Gegenstand des wirtschaftswissenschaftlichen Denkens in Wealth of Nations von Adam Smith (1776). Die Formulierung des Produktionsprozesses ausgehend von den zu tätigenden Aufgaben, unabhängig ob diese durch menschliche oder maschinelle Arbeit, durch Handel zugekauft oder an einem beliebigen Ort durchgeführt werden, ist dabei grundlegend im ökonomischen Verständnis verankert. Trotz dieser bereits recht alten Überlegungen haben die Computerisierung und die Digitalisierung dazu geführt, dass der durch sie ausgelöste technische Fortschritt sowie insbesondere die implizierten Folgen für Beschäftigung und Löhne in der aktuellen wirtschaftswissenschaftlichen Literatur nun differenzierter analysiert werden und in den vergangenen zwei Dekaden entsprechende formale M odelle entwickelt worden sind.

Mit dem vorliegenden Aufsatz möchte ich eine Einordnung des technischen Fortschritts durch die Computerisierung und Digitalisierung geben. Hierzu will ich kurz auf die Entwicklungsgeschichte des Computerzeitalters eingehen, um Gemeinsamkeiten und Unterschiede zu anderen Entwicklungen zu identifizieren. Im Anschluss gebe ich einen Überblick über den Erkenntnisstand zu den Effekten auf Einkommen und Beschäftigung auf Grundlage der verfügbaren Literatur, um die Erklärungen und Begründungen zu diskutieren.
\end{abstract}

\footnotetext{
${ }^{*}$ Dieser Aufsatz ist eine deutlich erweiterte und überarbeitete Fassung meines Referats für die 38. Tagung des Ausschusses für die Geschichte der Wirtschaftswissenschaften im Verein für Socialpolitik (Siegen, 08. bis 10. Juni 2017). Ich danke den Teilnehmerinnen und Teilnehmern für die vielfältigen wertvollen Hinweise und Anregungen. M ein weiterer Dank gilt Eva Barlösius, Knut Gerlach, Olaf Hübler, Fulko Lenz, Anna-Katharina Pikos und Andreas Wagener für die kritische Diskussion.

${ }^{\dagger}$ Kontaktadresse für Hinweise und Kritik: Prof. Dr. Stephan Thomsen, Institut für Wirtschaftspolitik, Leibniz Universität Hannover, Königsworther Platz 1, 30167 Hannover, Tel. 0511-762-14596, Fax. 0511-762-4574, Email: thomsen@wipol.uni-hannover.de.
} 


\section{Einleitung}

Die Entwicklung der Computer und die darauf aufbauende Digitalisierung haben technologische Veränderungen ausgelöst, die die Anforderungen an und die Nachfrage nach Arbeit, aber auch das Arbeitsangebot in den letzten Dekaden stetig verändert haben. Über die sechs Jahrzehnte seit ihrer Einführung in den 1950er Jahren hat sich die Computertechnologie durch eine rasante Entwicklung zu einer allgemein verfügbaren Technologie entwickelt und dabei alle Lebensbereiche erfasst. Als M eilensteine ihrer Evolution sind die Verbreitung der Heimcomputer (Personal Computer) in den 1980er Jahren, die Ausbreitung des Internets in den 1990er und insbesondere 2000er Jahren sowie davon ausgehend die allgemeine Digitalisierung in den letzten zehn Jahren zu nennen. ${ }^{1}$ Inwiefern diese technologischen Entwicklungen evolutionär oder revolutionär und damit vergleichbar zur industriellen Revolution im ausgehenden 18. und beginnenden 19. Jahrhundert sind, wird allerdings kontrovers diskutiert.

Unabhängig von dieser Diskussion ergeben sich aus der "Computerisierung" eine Reihe ökonomisch relevanter Fragen. Ein Schwerpunkt dieser Fragen liegt auf den Änderungen der Wertschöpfungs- und Produktionsprozesse durch veränderte Technologien, und hierbei insbesondere in den Effekten auf den menschlichen und maschinellen Faktoreinsatz und der entsprechenden Faktorproduktivität. In der ökonomischen Literatur der vergangenen rund 30 Jahre ist dies zunehmend eine empirische Debatte. ${ }^{2}$ Rückblickend betrachtet zeigen die bisher verfügbaren ökonomischen Analysen ein recht heterogenes Bild. Verbesserte Methoden und umfangreiche Daten haben zwar in jüngerer Zeit die Entwicklung von Modellen zur Beschreibung der Entwicklungen insbesondere auf dem Arbeitsmarkt ermöglicht. ${ }^{3}$ Bis heute ist jedoch kein Modell in der Lage, länger anhaltende Entwicklungen umfassend zu beschreiben bzw. zu erklären oder gar zukünftige Entwicklungen überzeugend zu prognostizieren. Insbesondere die Schwierigkeiten, den technischen Fortschritt, der durch die Digitalisierung entsteht, zu verstehen und inn außerdem geeignet zu messen, haben hierzu nicht unerheblich beigetragen.

Tatsächlich sind die betrachteten Fragen aber viel älter und finden sich bereits bei den klassischen Autoren. ${ }^{4}$ So erörtert Adam Smith in seinen Ausführungen zur Arbeitsteilung in „Wealth of Nations"

\footnotetext{
${ }^{1}$ Digitalisierung kann definiert werden als die auf digitale Weise stattfindende Transformation und Darstellung von Information und Kommunikation oder digitale M odifikation von Instrumenten und M aschinen.

${ }^{2}$ Dies liegt auch daran, dass die Disziplin zunehmend empirisch arbeitet. Diese Entwicklung ist ebenfalls als Ergebnis der Computerisierung und Digitalisierung zu sehen, da sie die Erfassung und Verarbeitung größerer Datenmengen ermöglicht hat.

${ }^{3}$ Beispielhaft seien hier die Arbeiten von Autor et al. (2003), Goos and M anning (2007) und Acemoglu und Autor (2011) genannt; auf diesen Arbeiten setzt eine umfangreiche, vor allem empirische Literatur zur Untersuchung des digitalen Wandels auf die Arbeitswelt, auf den internationalen Handel, auf die Produktion und auf weitere ökonomische Aspekte auf.

${ }^{4}$ Bereits Aristoteles diskutiert in seiner "Politik" die potenzielle Ersetzbarkeit menschlicher und maschineller Arbeit. Im 1. Buch, Kapitel 4 heißt es: „Von diesen Werkzeugen sind nun welche leblos, andere lebendig; so ist für
} 
(WN, Smith 1776) ausführlich die Entwicklung und den Einsatz von M aschinen und damit auch die Bedeutung von Innovationen in der Wertschöpfung. Die Effizienzgewinne sieht er hierbei als grundsätzlich positiv für Wirtschaft und Gesellschaft an. David Ricardo beurteilt die Entwicklung und den Einsatz von Maschinen in "On the Principles of Political Economy and Taxation“ (Principles, Ricardo 1821) ebenfalls als etwas im Allgemeinen Positives. Er erkennt und benennt aber auch die möglichen negativen Folgen für die Beschäftigten und führt Überlegungen zur Entstehung einer Art technologischer Arbeitslosigkeit an. Diese tritt auf, wenn die menschliche Arbeitskraft schneller durch Maschinen ersetzt wird als anderswo in der Wirtschaft neue Nachfrage nach (dieser freigesetzten) Arbeit entsteht. M arx führt im „Kapital“ (1867) diesen Punkt weiter aus und erörtert ausführlich nachteilige, aber auch kompensatorische Effekte der technologischen Entwicklung durch die wachsende Mannigfaltigkeit der gesellschaftlichen Produktionszweige. ${ }^{5}$ In ähnlicher Weise erklärt Keynes mehr als ein Jahrhundert nach Ricardo in der "General Theory“ (Keynes 1936) die M öglichkeit anhaltender Unterbeschäftigung durch die Herausstellung der Bedeutung der effektiven Nachfrage.

Die technologischen Veränderungen, die mit der M assencomputerisierung seit den 1970er Jahren einhergehen, haben sich unterschiedlich auf die verschiedenen Arbeitsqualitäten ausgewirkt. Die relativen Bildungsrenditen, d.h. die (erwarteten) Lohnaufschläge für Personen mit höheren Qualifikationen im Vergleich zu Personen mit geringeren Qualifikationen, sind seit den 1970er Jahren und mindestens bis zur Jahrtausendwende in den entwickelten Volkswirtschaften stark gestiegen. Seit den 1980er Jahren stieg außerdem die Lohnungleichheit zunächst in den USA, insbesondere getrieben durch höhere Löhne für besser qualifizierte Beschäftigte (Katz und M urphy, 1992). Gleichzeitig weitete sich aber auch das Arbeitsangebot dieser besser qualifizierten Beschäftigten deutlich aus.

Die wichtigste Erklärung für den Lohnanstieg trotz dieser Beschäftigungsausweitung war zunächst ein faktorverzerrter technischer Fortschritt. Dieser ist komplementär zu den Fähigkeiten der besser

den Steuermann das Steuerruder das leblose und der Untersteuermann am Vordertheil des Schiffes das lebendige Werkzeug; denn der Gehülfe ist in den Gewerben eine Art von Werkzeug. So ist also das einzelne Besitzstück ein Werkzeug zum Leben und das Vermögen eine M enge von Werkzeugen und der Sclave ein lebendiges Besitzstück und jeder Diener ein Werkzeug statt vieler. Denn wenn es möglich wäre, dass jedes Werkzeug auf Geheiss oder vorbewusst sein Werk vollbringen könnte, wie angeblich die Statuen des Dädalos oder die Dreifüsse des Hephästos, von denen der Dichter sagt, dass sie von selbst sich in die Versammlung der Götter begeben hätten und wenn so auch das Weberschiff von selbst webte und die Zither von selbst spielte, so bedürften weder die Künstler der Gehülfen, noch die Herren der Sclaven." (Aristoteles, 1880)

${ }^{5}$ Schöne Beispiele dazu aus dem Kapital, Bd. 1, Vierter Abschnitt, Kapitel 13.6 (M arx, 1968): „Ergreift die M aschinerie Vor- und Zwischenstufen, welche ein Arbeitsgegenstand bis zu seiner letzten Form zu durchlaufen hat, so vermehrt sich mit dem Arbeitsmaterial die Arbeitsnachfrage in den noch handwerks- oder manufakturmäßig betriebnen Gewerken, worin das M aschinenfabrikat eingeht. “ (S. 468) „Entsprechend der steigenden Masse von Rohstoffen, Halbfabrikaten, Arbeitsinstrumenten usw., die der Maschinenbetrieb mit relativ geringer Arbeitszahl liefert, sondert sich die Bearbeitung dieser Rohstoffe und Halbfabrikate in zahllose Unterarten, wächst also die M annigfaltigkeit der gesellschaftlichen Produktionszweige. Der M aschinenbetrieb treibt die gesellschaftliche Teilung der Arbeit ungleich weiter als die M anufaktur, weil er die Produktivkraft der von inm ergriffnen Gewerbe in ungleich höhrem Grad vermehrt." (S. 469) 
Qualifizierten und steigert dadurch ihre Produktivität sowie die Nachfrage nach ihren Qualifikationen; zugleich substituiert er die Fähigkeiten der schlechter Qualifizierten (Skill-biased Technological Change Hypothesis, SBTC, siehe Acemoglu (2002), Aghion (2002) oder Katz und Autor (1999)). Theoriegeschichtlich löste die Formulierung der SBTC-Hypothese den faktorneutralen technischen Fortschritt ab. Solow (1957) hatte mit dem Konzept der totalen Faktorproduktivität (TFP) technischen Fortschritt als faktorneutral angenommen: die TFP war (ist) ein Anstieg im Output, bei dem die Grenzrate der Transformation für gegebene Inputs unverändert bleibt. Die beobachteten Änderungen der relativen Beschäftigtenqualitäten verbunden mit den Preisanstiegen seit den 1970er Jahren konnten jedoch nicht durch Bewegungen entlang der Produktionsfunktion erklärt werden. Ein faktorneutraler technischer Fortschritt (wie die TFP) kann die Änderungen der relativen Preise daher nicht in befriedigender Weise erklären.

Ließen sich die divergierenden Entwicklungen der Arbeitsnachfrage nach unterschiedlichen Beschäftigungsqualitäten der 1970er und 1980er unter Verwendung der SBTC-Hypothese gut charakterisieren, hat sich insbesondere in den 1990er und 2000er Jahren ein abweichendes Phänomen herauskristallisiert: Die Arbeitsnachfrage stieg nun nicht mehr nur am oberen Ende der Qualifikationsverteilung, sondern auch am unteren Ende. Damit einhergehend verringerte sich die Zahl mittlerer Qualifikationen und es kam zu einer Art Aushöhlung des Arbeitsmarkts (Polarisierung, siehe z.B. Autor et al. (2003), Goos und Manning (2007), Goos et al. (2009)). Um diese Entwicklungen zu erklären, änderte sich das methodische Vorgehen der Analysen basierend auf den Überlegungen von Autor et al. (2003). Standen bisher immer die formalen Qualifikationen der Individuen im Mittelpunkt der Bestimmung von Bildungsrenditen (das sogenannte Lehrbuchmodell oder, wie Acemoglu und Autor (2011) es nennen, das kanonische M odell, basierend u.a. auf den Ideen von Becker (1993), Ben-Porath (1967) und M incer (1974) mit einer Fokussierung auf die Eigenschaften/Fähigkeiten der Arbeitsanbieter), bekamen nun die Tätigkeiten, die mit diesen Fähigkeiten ausgeführt werden können, zentrale Bedeutung. ${ }^{6}$ Anders ausgedrückt wird der Nachfrage nun mehr Gewicht beigemessen.

Dieser Tätigkeiten-basierte Ansatz (task-based approach, Autor et al. (2003) und Acemoglu und Autor (2011)) charakterisiert die Tätigkeiten nach ihrem Potenzial, durch Computer ersetzbar zu sein. Hierbei sind sogenannte Routinetätigkeiten, d.h. sich wiederholende Arbeitsgänge, die standardisiert und damit programmierbar sind, besonders leicht durch Computer zu ersetzen. Nicht-Routinetätigkeiten, die aus menschlicher Sicht nicht zwingend schwieriger, aber aus technischer Sicht komplexer sind, können hingegen nur sehr viel schwieriger durch Computer substituiert werden. Die Besonderheit die-

\footnotetext{
${ }^{6}$ Acemoglu und Autor (2011) bezeichnen den von innen vorgeschlagenen Ansatz als ricardianisch, da Beschäftigte über unterschiedliche komparative Vorteile in der Erstellung von Gütern und Dienstleistungen verfügen.
} 
ser Klassifizierung der Tätigkeiten liegt darin, dass nicht-Routinetätigkeiten sowohl bei sehr qualifizierten Berufen (z.B. Managern), aber auch bei Berufen mit geringen Qualifikationsanforderungen und vielen unterschiedlichen Aufgaben (besonders im Bereich der Dienstleistungen, z.B. Servicekräfte, Pflegekräfte) anfallen. Routinetätigkeiten sind hingegen charakteristisch für eine Vielzahl von qualifizierten Berufen, insb. mit mittleren Qualifikationen (z.B. Buchhalter, Sachbearbeiter). Diese Tätigkeiten konkurrieren daher in besonderer Weise mit den Möglichkeiten digitaler Ersetzbarkeit. Goos et al. (2009) bezeichnen den zugrundeliegenden technischen Fortschritt entsprechend als Routine-biased Technological Change (RBTC), da er bezüglich routinierter Tätigkeiten verzerrt ist.

Der Tätigkeiten-basierte Ansatz kann die beobachtete Polarisierung der Arbeitsmärkte der vergangenen zwei Dekaden deutlich besser erklären als die SBTC-Hypothese. Inwieweit bzw. wie lange die RBTC-Hypothese in der Lage sein wird, den fortschreitenden Beschäftigungswandel aufgrund der Weiterentwicklungen der Computer- und Digitaltechnik und ihrer Folgen für alle Formen und Qualitäten der Beschäftigung zu erklären, ist eine offene Frage. Gerade die jüngste empirische Evidenz deutet darauf hin, dass sich die Polarisierung nicht stetig fortsetzt bzw. bereits eine Umkehr begonnen hat, da die bald gänzlich erfolgte Digitalisierung für noch bestehende Routinetätigkeiten wieder an Bedeutung gewinnt; nun allerdings in komplementärer und nicht mehr nur substituierender Form (Autor et al., 2014).

Der Tätigkeiten-basierte Ansatz folgt - auch wenn von den Autoren nicht herausgestellt - im Kern der schon von Adam Smith in WN (Smith 1776) formulierten Arbeitsteilung, in der er zunächst die Unterteilung des Wertschöpfungsprozesses in einzelne, spezialisierte Arbeitsschritte mit deutlich gesteigerter Produktivität trotz geringer Komplexität bzw. Anforderungen an den einzelnen Arbeiter erläutert. ${ }^{7}$ Er setzt seine Ausführungen aber noch fort, indem er die Entwicklung und den Einsatz von M aschinen explizit am Ersatz bestimmter Tätigkeiten in diesem arbeitsteiligen Prozess erläutert. Diese führen dann zu einer weiteren Steigerung der Produktivität bei gleichzeitiger Senkung des individuelIen Arbeitsleid. ${ }^{8}$ D.h. bereits für Smith ist es unerheblich, ob M aschine oder M ensch die Tätigkeit vollziehen.

\footnotetext{
${ }^{7}$ Hier sei auf das berühmte Stecknadelbeispiel zur Arbeitsteilung verwiesen. Durch Aufteilung der Tätigkeiten in sehr einfache Arbeitsschritte kann mit ungelerntem Personal ein Vielfaches von dem erzeugt werden, was selbst ein geübter Arbeiter schaffen könnte. Smith sieht dabei zwar zeitbedingt Grenzen der Übertragbarkeit seiner Analogie auf bestimmte Bereiche, formuliert aber dennoch in grundsätzlicher Weise: „In every other art and manufacture, the effects of the division of labour are similar to what they are in this very trifling one; though, in many of them, the labour can neither be so much subdivided, nor reduced to so great a simplicity of operation. The division of labour, however, so far as it can be introduced, occasions, in every art, a proportionable increase of the productive powers of labour." (Smith 1776, Book 1, Ch. 1, par. 4)

${ }^{8}$ Ein schönes Beispiel hierzu ist die Weiterentwicklung einer Dampfmaschine durch einen Jungen, der die Ventile steuern sollte: "In the first fire-engines, a boy was constantly employed to open and shut alternately the communication between the boiler and the cylinder, according as the piston either ascended or descended. One of those boys, who loved to play with his companions, observed that, by tying a string from the handle of the valve
} 
Der Tätigkeiten-basierte Ansatz greift aber noch auf weitere Überlegungen zurück. Durch die Unterscheidung von Tätigkeiten anhand ihres Substitutionsgrads impliziert er zugleich eine unterschiedliche Betroffenheit möglicher Produktivitätseffekte durch die Digitalisierung in verschiedenen Berufen. Offenkundig können dabei besser bzw. leichter digitalisierbare Bereiche der Wirtschaft eher von möglichen Produktivitätszuwächsen profitieren als solche, die nur wenig routiniert und damit schwerer zu ersetzen sind. Ähnliche Gedanken finden sich bereits bei Baumol (1967, 2013). Gerade die jüngeren Beschäftigungsentwicklungen, die auf eine Umkehr der Polarisierung hinweisen, sollten entsprechend in Zusammenhang mit Baumols Kostenkrankheit gesehen werden. Baumol (1967) analysiert den Einfluss progressiver und stagnierender Sektoren auf das Wirtschaftswachstum. Die stagnierenden Sektoren (insb. Dienstleistungen) werden vor allem durch personenbezogene Dienstleistungen charakterisiert. Diese sind durch den menschlichen Faktor in möglichen Produktivitätszuwächsen begrenzt und lassen sich aufgrund der Art der auszuführenden Tätigkeiten daher auch nicht oder nur schwerlich automatisieren. In diesen Sektoren fällt der Produktivitätsfortschritt entsprechend geringer aus als in den progressiven Sektoren (insb. der Industrie). Unter der Annahme der langfristigen Angleichung der Löhne über alle Sektoren hinweg steigen die Lohnstückkosten und Preise der Dienstleistungen schneller im Vergleich zu denen für Industriegüter. Daher verteuern sich die Dienstleistungen relativ gegenüber den Industriegütern und der Anteil der Beschäftigten in produktivitätsschwachen Dienstleistungssektoren steigt immer weiter, was letztlich zu einer Verringerung des gesamtwirtschaftlichen Wachstums führt (Stagnationstheorie). Darüber hinaus hängt die Entstehung bzw. Vermeidung von personeller Lohnungleichheit entscheidend von den tatsächlichen Lohnsteigerungen in den nicht-automatisierbaren im Vergleich zu den automatisierbaren Sektoren ab. Die beobachteten Entwicklungen einer (zumindest vorübergehend) zunehmenden Ungleichheit können daher Folge asymmetrischer Produktivitätszuwächse in den einzelnen Sektoren der Volkswirtschaft sein.

Begleitet wird die gesellschaftliche und wissenschaftliche Diskussion der Digitalisierung durch die Furcht vor möglicher technologischer Arbeitslosigkeit und wachsender Ungleichheit. Dies zeigen gerade jüngere, viel beachtete Veröffentlichungen zu den möglichen Folgen für die Beschäftigung. Die Prognose von Frey und Osborne (2017) berechnet die Zahl potenziell digital ersetzbarer Arbeitsplätze in den USA: ihren Berechnungen zufolge arbeiten 47\% der Beschäftigten in Berufen, die in den nächsten zehn bis 20 Jahren automatisierbar sein sollten. ${ }^{9}$ Die zugrundeliegende Annahme der Ersetzbarkeit

\footnotetext{
which opened this communication to another part of the machine, the valve would open and shut without his assistance, and leave him at liberty to divert himself with his playfellows. One of the greatest improvements that has been made upon this machine, since it was first invented, was in this manner the discovery of a boy who wanted to save his own labour." (Smith 1776, Book 1, Ch. 1, par. 8)

${ }^{9}$ Auch dieser gesellschaftliche Diskurs dauert schon viel länger an. Bereits vor rund 40 Jahren, in der Ausgabe vom 17. April 1978, titelte der Spiegel: „Die Computer-Revolution: Fortschritt macht arbeitslos“.
} 
ganzer Berufe aufgrund überwiegender automatisierbarer Tätigkeitsanteile ist aber sehr kritisch zu sehen. Differenziertere Analysen auf der Grundlage von Tätigkeitsstrukturen von Bonin et al. (2015) und Arntz et al. (2016) sehen eher 9\% der Arbeitsplätze im gleichen Zeitraum als potenziell ersetzbar an.

Die Furcht bzw. negative Erwartung, dass Technik den Menschen verdrängt, ist nicht neu. Bereits Ricardo (1821) diskutiert die möglichen Nachteile für die Arbeiter, wenn ihre Arbeitsplätze durch Maschinen ersetzt würden und eine Beschäftigung in anderen Bereichen der Wirtschaft nicht erfolgte: dies geschehe genau dann, wenn die Nachfrage nach Gütern nicht in gleicher Weise steige wie die Produktivität. Die verfügbaren Berechnungen und die Diskussion über die negativen Folgen der Computerisierung und Digitalisierung lassen in weiten Teilen bisher allerdings die kompensatorischen Effekte der Digitalisierung außer Acht. Sie implizieren, dass die Produktivitätssteigerung eine effizientere Produktion erlaubt, die mit einer Umverteilung von Beschäftigung zwischen den Sektoren und Reallohnsteigerungen verbunden ist. Dies wiederum führt zu einem Anstieg der Nachfrage insgesamt. In einem solchen Fall kann der Fortschritt durch die Digitalisierung dann beschäftigungsneutral oder gar beschäftigungssteigernd sein (insofern Unterbeschäftigung bzw. Arbeitslosigkeit in der Ökonomie vorlag).

Nicht nur die aktuell diskutierten Gefahren der Digitalisierung ähneln also denen bereits in der industriellen Revolution diskutierten. Auch die Ideen, Überlegungen und Empfehlungen der Klassiker sollten in der aktuellen Diskussion berücksichtigt werden. So macht bereits Ricardo deutlich, dass trotz der möglichen negativen Effekte für die Beschäftigten ein Verbot des Fortschritts kaum förderlich ist. Den Fortschritt durch M aschinen bzw. bezogen auf die heutige Zeit durch die Digitalisierung zu verbieten, führe langfristig zu größeren Schäden für die Ökonomie, da Kapital mobil ist und die Vorteile dann in anderen Ländern generiert werden.

Mit dem vorliegenden Aufsatz möchte ich drei Dinge erreichen: zuerst möchte ich eine Einordnung des technischen Fortschritts durch die Computerisierung und Digitalisierung geben. Hierzu will ich die Entwicklungsgeschichte des Computerzeitalters kurz beschreiben, um Gemeinsamkeiten und Unterschiede zu anderen Entwicklungen zu identifizieren. Im Anschluss gebe ich einen Überblick über den Erkenntnisstand zu den Effekten auf Einkommen und Beschäftigung auf Grundlage der verfügbaren Literatur, dem eine Diskussion der Erklärungen und Begründungen folgt. Am Ende werden Überlegungen zu politischen Implikationen aufgrund entstehender Ungleichheiten angesprochen.

\section{Technischer Fortschritt: Entstehung und Folgen}

\subsection{Wie entsteht technischer Fortschritt?}

Bevor die Frage beantwortet werden kann, wie Computer und Digitalisierung den technischen Fortschritt bestimmen und dadurch die Produktion von Waren und Dienstleistungen verändern, erscheint 
mir eine kurze, grundlegende Beschreibung des Innovationsprozesses sinnvoll. Bereits Adam Smith macht den technischen Fortschritts zum Gegenstand des ökonomischen Denkens. In seinen Ausführungen zur Arbeitsteilung (Smith 1776, Book 1) geht er auf die drei bestimmenden Ursachen ein. Neben Spezialisierung bzw. steigender Arbeitsproduktivität durch Lerneffekte sowie der Verringerung der Rüstzeiten durch Arbeitsteilung sind die Entwicklung und der Einsatz von M aschinen entscheidend:

"I shall only observe, therefore, that the invention of all those machines by which labour is so much facilitated and abridged seems to have been originally owing to the division of labour. Men are much more likely to discover easier and readier methods of attaining any object when the whole attention of their minds is directed towards that single object than when it is dissipated among a great variety of things. But in consequence of the division of labour, the whole of every man's attention comes naturally to be directed towards some one very simple object. It is naturally to be expected, therefore, that some one or other of those who are employed in each particular branch of labour should soon find out easier and readier methods of performing their own particular work, wherever the nature of it admits of such improvement. A great part of the machines made use of in those manufactures in which labour is most subdivided, were originally the inventions of common workmen, who, being each of them employed in some very simple operation, naturally turned their thoughts towards finding out easier and readier methods of performing it." (Smith 1776, Book 1, Ch. 1, par. 8)

Die Arbeitsteilung ist also nicht nur Ergebnis, sondern in besonderer Weise durch die mit ihr verbundene Spezialisierung und Fokussierung auf einzelne Arbeitsschritte Ursache der Entwicklung und Verbreitung von M aschinen im Arbeitsprozess. Der so formulierte Einsatz der M aschinen beschreibt im engeren Sinne aber nur Prozessinnovationen, d.h. Produktivitätszuwächse in der Erstellung bestimmter, festgelegter Produkte. Dennoch führt dieser Prozess auch zu Produktinnovationen, zumindest im Bereich der Maschinenentwicklung, da sich der Maschinenbau zu einem eigenen Sektor entwickelt hat:

"All the improvements in machinery, however, have by no means been the inventions of those who had occasion to use the machines. Many improvements have been made by the ingenuity of the makers of the machines, when to make them became the business of a peculiar trade." (Smith 1776, Book 1, Ch. 1, par. 9)

Eigentlicher Raum für Produktinnovationen ergibt sich in diesem Gedankengerüst zugleich nur beschränkt. Dies bleibt auch bei David Ricardo so, der in den Principles (Ricardo 1821, insb. Ch. 31) zwar den Nutzen bzw. Schaden durch den Einsatz von M aschinen erkennt, aber nicht erklärt, wie es zu wirklichen Produktinnovationen kommt. Einen großen Beitrag, um dieses zu verstehen und zu erklären, leistet Schumpeter. In seiner Habilitationsschrift von 1911 (siehe Schumpeter 1997) setzt er sich mit der Entstehung von Innovationen auseinander. Er sieht wirtschaftliches Wachstum durch die Erstellung „neuer Kombinationen“ als entscheidend an. Diese gehen dabei über den „statischen“ Fortschritt der Wirtschaft hinaus und schaffen die notwendige "Dynamik" durch eine Störung des statischen Gleichgewichts. In seiner Untersuchung möchte er dabei die Frage beantworten: „Wie vollziehen sich solche Veränderungen, und welche wirtschaftlichen Erscheinungen lösen sie aus?“ (Schumpeter, 1997, S. 94). 
Eine entsprechende Veränderung ist für Schumpeter dann gegeben, wenn sie "nicht vorkommt unter den Entscheidungen des Kreislaufs oder der Gleichgewichtstendenz, sondern nur wie eine äußere M acht in sie hineinwirkt." (Schumpeter 1997, S. 98). Aber nicht jede solche Veränderung des Kreislaufs oder Verschiebung der Gleichgewichtstendenz erzeugt die erforderliche Dynamik, sondern nur solche, die spontan der Wirtschaft entspringen und zweitens diskontiunierlich sind. ${ }^{10} \mathrm{Er}$ bezeichnet seine Überlegungen entsprechend auch als „Theorie des Übergangs der Volkswirtschaft von dem jeweils gegebenen Gravitationszentrum zu einem andern“ (Schumpeter 1997, S. 99). Die neuen Kombinationen umfassen dabei fünf verschiedene Fälle: 1) die Herstellung eines neuen Gutes oder einer neuen Qualität, 2) die Einführung einer neuen Produktionsmethode, 3) die Erschließung eines neuen Absatzmarktes, 4) die Eroberung einer neuen Bezugsquelle von Rohstoffen und Vorprodukten sowie 5) die Durchführung einer Neuorganisation.

Hiermit definiert er den Raum möglicher Innovationen sehr weit, da die neuen Kombinationen im Wesentlichen über ihren Impuls für das wirtschaftliche Gleichgewicht definiert werden und weniger inr enger(er) Charakter von Bedeutung ist. Es ist also nicht das Produkt als solches (bzw. einer der vier übrigen Fälle), das entscheidend ist, sondern erst die von ihm ausgehende Veränderung der Volkswirtschaft charakterisiert die Innovation. Dies bedeutet andersherum, dass es auch neue Kombinationen gibt, die zu diesem Impuls nicht fähig sind. Hierzu zählen zum einen Entwicklungen, die lediglich einen nach Schumpeter statischen Fortschritt der Wirtschaft bedingen, und selbstverständlich solche, die gar nicht zur Anwendung kommen.

Weitzman (1998) beschreibt den Innovationsprozess in ähnlicher Weise als Kombination bereits bestehender Ideen, der dadurch neue Ideen hervorbringt. Von diesen neuen bzw. kombinierten Ideen sind wiederum aber nur wenige brauchbar und noch weniger werden in brauchbare Form übertragen. Sehr anschaulich illustriert er seine Überlegungen am Beispiel der Erfindung der Glühbirne durch Edison: auch sie ist im Wesentlichen eine Kombination vieler Bestandteile, die bereits erfunden bzw. entwickelt waren. Der wesentliche Clou war die Entwicklung bzw. Entdeckung eines brauchbaren Glühfadens. Um den geeigneten kohlenstoffhaltigen Grundstoff zu finden, ließ Edison mehr als 6.000 organische $M$ aterialen auf ihre Brauchbarkeit hin durch seine $M$ itarbeiter überprüfen, bis sich der geeignete schließlich als japanischer Bambus herausstellte. Edison beschrieb diesen Innovationsprozess selbst mit dem Begriff "Ringfahndung“. Diese Beschreibung hebt zugleich das Wesen des Innovationsprozesses klar hervor. Nicht die Ideen stellen die Beschränkungen dar, sondern ihre Verarbeitung, Umsetzung

\footnotetext{
${ }^{10}$ Als Störungsursachen unterscheidet er zwischen fünf hauptsächlichen Gründen: 1) die Zunahme des Kapitals, 2) die Zunahme der Bevölkerung, 3) Veränderungen der Geschmacksrichtungen der Konsumenten (Präferenzen), 4) Veränderungen der Technik und 5) Änderungen in der Organisation der Produktion. Während die ersten drei Ursachen gleichermaßen auch für den statischen Fortschritt, d.h. die kontinuierliche Entwicklung gelten, sind insbesondere die letzten beiden für die Erstellung der neuen Kombinationen wesentlich.
} 
und Durchsetzbarkeit. Weitzmans M odell geht auf eben diese letzten beiden Punkte ein: die Beschränkung des Wachstums liegt nicht so sehr in der Fähigkeit der Formulierung von wirklich neuen Ideen, sondern vielmehr in der Fähigkeit, die geradezu unerschöpflichen Kombinationen neuer Ideen in brauchbare Form zu übertragen.

Aus der Charakterisierung als Kombination wird bei Schumpeter (1997) zugleich deutlich, dass die Innovation auf Bestehendes zurückgreift. Hierbei wird sie aber nicht zwingend von den Eigentümern der alten Kombinationen entwickelt bzw. erkannt:

„[E]s kann erstens vorkommen, aber es gehört nicht zum Wesen der Sache, dass die neuen
Kombinationen von denselben Leuten durchgesetzt werden, welche den Produktionsprozess o-
der den kommerziellen Weg der Waren in jenen eingelebten alten Kombinationen beherrschen,
die durch die neuen überholt und verdrängt werden. Vielmehr treten der Idee und auch der
Regel nach die neuen Kombinationen, bzw. die sie verkörpernden Firmen, Produktionsstätten
usw., nicht einfach an die Stelle, sondern zunächst neben die alten, die aus sich heraus meist
gar nicht in der Lage wären, den neuen großen Schritt zu tun: es waren [...] im Allgemeinen
nicht die Postmeister, welche die Eisenbahnen gründeten." (Schumpeter 1997, S. 101)

$M$ it dieser Beschreibung bereitet er eine weitere, wesentliche Anforderung für das Entstehen neuer Kombinationen vor: die Unternehmereigenschaft. Er unterscheidet den Unternehmer vom Kapitalisten und vom Betriebsleiter oder Wirt, insbesondere durch seine M otive für die Erstellung neuer Kombinationen. Der Unternehmer hat für inn zentrale Bedeutung in der Erstellung der neuen Kombinationen und damit der Veränderung des volkswirtschaftlichen Gleichgewichts. Unternehmer ist derjenige, der die neue Kombination durchsetzt. ${ }^{11}$ Diese Eigenschaft ist zugleich vergänglich: durch die Innovation kann der Unternehmer einen Monopolgewinn realisieren, der durch das Auftreten von Nachahmern über die Zeit aufgezehrt wird. Nur das stetige Entwickeln und Durchsetzen weiterer neuer Kombinationen sichert den dynamischen Unternehmer ab. ${ }^{12}$ Gleichzeitig ist die zentrale M otivation des Unternehmers nicht allein seine materielle Bedürfnisbefriedigung, ein bestimmtes Nutzenkalkül oder besondere Gier nach monetären Erträgen; für Schumpeter folgen Unternehmer - da die finanzielle Bedürfnisbefriedigung schnell gesichert ist - vielmehr psychologischen M otiven, wie z.B. der Traum und Wille zur Begründung eines eigenen Reichs, der Siegerwille oder auch die Freude am Gestalten.

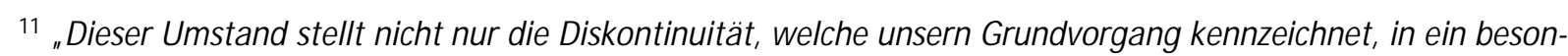
deres Licht, schafft sozusagen zur ersten früher dargelegten noch eine zweite Diskontinuität, sondern er beherrscht auch den Verlauf der Begleiterscheinungen." (Schumpeter 1997, S. 101)

12 In Abgrenzung zum Unternehmer finanziert der Kapitalist die Entwicklung und Durchsetzung neuer Kombinationen und trägt das entsprechende Risiko. Der Betriebsleiter oder Wirt hingegen ist derjenige Unternehmer, der ohne neue Kombinationen durchzusetzen lediglich zur statischen Weiterentwicklung der Wirtschaft beiträgt und entsprechend einen Normalgewinn generiert. Übergänge zwischen den verschiedenen Gruppen sind möglich.
} 


\subsection{Folgen des technischen Fortschritts}

Da die neuen Kombinationen also zunächst neben und später an die Stelle der bestehenden alten Kombinationen treten, erzeugen sie in klarer Weise Wettbewerb um die Ressourcen. Die Folge des Fortschritts ist also Konkurrenz zur bestehenden Situation:

„In nur teilweisem Zusammenhang mit diesem Moment ist [...] zu beachten, dass wir uns die Durchsetzung der neuen Kombinationen und das Entstehen ihrer Verkörperungen grundsätzlich niemals so vorzustellen haben, wie wenn sie ungenützte Produktionsmittel in sich vereinigten." (Schumpeter 1997, S. 102)

Wesen der neuen Kombination ist damit die Verwendung bereits bestehender Produktionsmittel, die bisher für andere Zwecke genutzt worden sind:

„In der Regel muss die neue Kombination die Produktionsmittel, die sie braucht, irgendwelchen alten Kombinationen entziehen - und aus den erwähnten Gründen können wir sagen, dass sie das grundsätzlich immer tut. [...] Die Durchsetzung neuer Kombinationen bedeutet also [...] Andersverwendung des Produktionsmittelvorrats der Volkswirtschaft." (Schumpeter 1997, S. 102f.)

Verbunden mit der von Schumpeter gemachten Analogie, dass auch nicht die Postmeister die Eisenbahnen gründeten (s.o.), impliziert dies, dass in der Regel neue Unternehmer auf den Plan treten und als klare Folge des Fortschritts damit Konkurrenz zwischen den Unternehmen entsteht. Kurz (2017, S. 786) schreibt hierzu treffend: „Innovationen sind nach Joseph A. Schumpeter Prozesse ,schöpferischer Zerstörung'. Sie rufen Neues ins Leben und vernichten Altes. Sie kennen nicht nur Gewinner, sondern auch Verlierer." Die Suche nach neuen Kombinationen wird also durch die Konkurrenz zusätzlich angefacht, da die Unternehmen die Innovation benötigen, um Wettbewerbsvorteile zu generieren. Die neuen Kombinationen stellen wiederum einen technischen Fortschritt dar, der Produktivitätszuwächse impliziert. Die höhere Produktivität führt zu effizienteren Kostenstrukturen und möglichen niedrigeren Preisen, wodurch sich die Wettbewerbssituation verbessert. Dieser Prozess setzt sich dabei entsprechend dynamisch fort, da für die Unternehmen kontinuierliche Anreize zum Auffinden neuer Kombinationen bestehen.

Dies ist zugleich die zentrale Überlegung zur Weiterentwicklung der Theorie durch Schumpeter. Er kritisiert die „überkommene Theorie“ (Schumpeter 1997, S. 106) dafür, dass sie das Vorhandensein der Produktionsmittel für neue Entwicklungen nicht erklären kann. Sein dynamischer Ansatz hat diese Einschränkung nicht:

„Es existiert nicht im Kreislauf, weil dessen Phasen nur auf Grund schon vorhandener Produktionsmittelmengen ablaufen und deren Entstehen nicht aus ihm selbst erklärt werden kann. Es existiert nicht für die Durchsetzung neuer Kombinationen, weil diese die Produktionsmittel, die sie brauchen, dem Kreislauf entnehmen, wobei es völlig einerlei ist, ob sie diese M ittel im Kreislauf schon so vorfinden, wie sie sie brauchen, wie vor allem die, ursprünglichen' und besonders die unqualifizierte Handarbeit, oder erst herstellen oder herstellen lassen müssen, wie viele der ,produzierten' Produktionsmittel." (Schumpeter 1997, S. 106) 
Nach Schumpeter führt der technische Fortschritt entsprechend zu einem Wachstum der Wirtschaft - auch wenn seine Theorie nicht nur Gewinner (die Durchsetzer der neuen Kombinationen), sondern auch Verlierer kennt (die Besitzer der alten Kombinationen). Durch die Umnutzung der Ressourcen kommt es in der Ökonomie aber nicht zu ungenützten Potenzialen, sondern schließlich zu einer effizienteren Nutzung und damit zu einem größeren Ergebnis.

Der technische Fortschritt ist damit als wichtige Quelle für Produktivitätssteigerungen und Wirtschaftswachstum identifiziert. Im Gegensatz zu anderen Wachstumsfaktoren, wie dem Lernkurveneffekt und der Humankapitalakkumulation, ermöglicht er unter der Annahme eines abnehmenden Grenznutzens dabei jedoch allein langfristiges Wachstum. Denn sowohl die Produktivitätsfortschritte durch Lerneffekte der Arbeiter als auch das Potenzial zur Aus- und Weiterbildung der Beschäftigten sind begrenzt und zusätzliche Investitionen mit abnehmenden Grenzerträgen verbunden.

Bereits Ricardo sah den technischen Fortschritt trotz - oder gerade wegen der Umverteilung - von Arbeit zu Kapital zunächst in ähnlicher Weise positiv:

„Ever since I first turned my attention to questions of political economy, I have been of opinion, that such an application of machinery to any branch of production, as should have the effect of saving labour, was a general good, accompanied only with that portion of inconvenience which in most cases attends the removal of capital and labour from one employment to another." (Ricardo 1821, Ch. 31.2)

Hierbei sollten alle gesellschaftlichen Gruppen vom technischen Wandel profitieren, also insbesondere auch die Beschäftigten, deren Arbeitsplätze zunächst rationalisiert, die dann aber für die Erstellung anderer Waren wiedereingestellt würden:

"The class of labourers also, I thought, was equally benefited by the use of machinery, as they would have the means of buying more commodities with the same money wages, and I thought that no reduction of wages would take place, because the capitalist would have the power of demanding and employing the same quantity of labour as before, although he might be under the necessity of employing it in the production of a new, or at any rate of a different commodity. [...] If, by improved machinery, with the employment of the same quantity of labour, the quantity of stockings could be quadrupled, and the demand for stockings were only doubled, some labourers would necessarily be discharged from the stocking trade; but as the capital which employed them was still in being, and as it was the interest of those who had it to employ it productively, it appeared to me that it would be employed on the production of some other commodity, useful to the society, for which there could not fail to be a demand." (Ricardo 1821, Ch. 31.2).

Insbesondere die erforderliche Entwicklung der Nachfrage nach den produzierten Gütern ließ Ricardo in der dritten Auflage der Principles seine Meinung aber revidieren. Wenn die Güternachfrage weniger stark steige als die Produktivität (durch die Maschinen bzw. den technischen Fortschritt), könnten die Beschäftigten ihre Arbeit aus technischen Gründen verlieren (Freisetzungstheorie). Er definiert damit eine mögliche nachfrageinduzierte technologische Arbeitslosigkeit, die später bei Keynes (1936) eine entscheidende Rolle spielen wird und auch in der aktuellen Diskussion um die Folgen der 
Computerisierung und Digitalisierung große gesellschaftliche Beachtung findet. In entsprechender Weise passt Ricardo seine optimistischen Aussichten bezüglich des technischen Fortschritts für die Beschäftigten an; seine Position bezüglich der Landbesitzer und Kapitalisten behält er aber bei:

"These were my opinions, and they continue unaltered, as far as regards the landlord and the capitalist; but I am convinced, that the substitution of machinery for human labour, is often very injurious to the interests of the class of labourers." (Ricardo 1821, Ch. 31.4)

Seine ursprüngliche Überlegung, dass wann immer das Nettoeinkommen der Ökonomie steigt, das Bruttoeinkommen in gleicher Weise steige, relativiert er entsprechend:

"I now, however, see reason to be satisfied that the one fund, from which landlords and capitalists derive their revenue, may increase, while the other, that upon which the labouring class mainly depend, may diminish, and therefore it follows, if I am right, that the same cause which may increase the net revenue of the country, may at the same time render the population redundant, and deteriorate the condition of the labourer." (Ricardo 1821, Ch. 31.4)

Im Weiteren diskutiert er die negativen Effekte der Einführung von Maschinen, die nicht aufgrund von Vorurteilen oder Fehlern abgelehnt werden, sondern aus rein ökonomischen Überlegungen den Arbeitern zum Nachteil gereichen können. Nach Ricardo ist die Einführung von Maschinen nur dann vorteilhaft für alle Klassen, wenn:

"[...] the improved means of production, in consequence of the use of machinery, should increase the net produce of a country in a degree so great as not to diminish the gross produce, (I mean always quantity of commodities and not value,) then the situation of all classes will be improved. The landlord and capitalist will benefit, not by an increase of rent and profit, but by the advantages resulting from the expenditure of the same rent, and profit, on commodities, very considerably reduced in value, while the situation of the labouring classes will also be considerably improved; 1st, from the increased demand for menial servants; 2dly, from the stimulus to savings from revenue, which such an abundant net produce will afford; and 3dly, from the low price of all articles of consumption on which their wages will be expended." (Ricardo 1821, Ch. 31.17).

Ricardo ist damit gewissermaßen unentschieden zwischen Freisetzungstheorie oder Kompensationstheorie. Beiden gemein ist, dass durch den technischen Fortschritt die Produktivität steigt. Nach der ersten Theorie steigt die Güternachfrage aber nicht unbedingt in gleicher Weise, was einen geringeren Arbeitseinsatz und damit Arbeitslosigkeit impliziert. Im Gegensatz besagt die Kompensationstheorie, dass nicht nur die Menge der produzierbaren Güter erhöht wird, sondern zugleich durch den technischen Fortschritt auch ihr Preis fallen muss. Der induzierte Realeinkommensanstieg, den Ricardo für alle Klassen sieht, führt dann wiederum zu einer Ausweitung des Konsums aller Güter; die erhöhte (Güter-)Nachfrage nach den übrigen Gütern impliziert eine höhere Arbeitsnachfrage. Der technische Fortschritt kann daher trotz Substitution von Beschäftigung in einzelnen Branchen insgesamt beschäftigungsneutral sein oder gar zu einer Erhöhung der Beschäftigung in der Gesamtwirtschaft, wenn die 
durch Rationalisierung frei gewordenen Beschäftigten in anderen Bereichen der Ökonomie wiedereingestellt werden. ${ }^{13}$ Übertragen auf die beobachteten Entwicklungen der Computerisierung und die Digitalisierung können sich also sowohl Risiken als auch Chancen für die Beschäftigung ergeben. Ob Chancen oder Risiken überwiegen, ist dabei eine empirische Frage. Die Unsicherheit ihrer Beantwortung befördert und erhitzt die gesellschaftliche Diskussion zugleich.

\section{Computer und Digitaltechnik: Eine zweite industrielle Revolution?}

\subsection{Ein kurzer Überblick über die Entwicklungsgeschichte}

Rechenmaschinen basierend auf Lochkarten wurden für Automatisierungsprozesse ${ }^{14}$ bereits kurz nach Beginn der industriellen Revolution entwickelt und eingesetzt. ${ }^{15}$ Diese analogen M aschinen wurden nach dem zweiten Weltkrieg von digitalen Rechenmaschinen Schritt für Schritt abgelöst. Das eigentliche Computerzeitalter beginnt in dieser Logik in den 1950er Jahren mit der Produktion und dem Vertrieb kommerzieller Computer. So bot z.B. IBM im Jahr 1955 mit dem Modell 608 den ersten vollständig transistorbasierten Rechner mit magnetischen Kernen für dynamische Speicherung an (Fierheller, 2014). ${ }^{16}$ Im Jahr 1958 erfasste das U.S. Bureau of Economic Analysis (BEA) zum ersten M al „Informationstechnologie" als eigene Investitionsgruppe (Brynjolfsson und M cAfee, 2015, S. 62). ${ }^{17}$

Die massenhafte Verbreitung von Computern setzte allerdings erst in den 1970er Jahren ein. Maßgeblich dafür war die Erfindung des Mikroprozessors (das erste Patent war der TM S1000, 1971 durch Texas Instruments), der im Gegensatz zu aufwändigen und großen Röhren nun halbleiterbasierte, kompakte Transistoren verwendete. ${ }^{18}$ Die höhere Verarbeitungsgeschwindigkeit durch eine höhere Taktfrequenz (Schaltgeschwindigkeit) wurde durch eine geringere Wärmeentwicklung und eine geringere

\footnotetext{
${ }^{13}$ Der Grad der Kompensation hängt von der volkwirtschaftlichen Produktionsfunktion, der Flexibilität der Preise und Löhne und der Geschwindigkeit des wirtschaftlichen Wachstums ab. Entsprechend wird eine ausreichend hohe Geschwindigkeit im Wachstum, bei dem Freisetzung und Einstellung von Arbeitskräften im Einklang sind, als kompensatorisches Wachstum bezeichnet.

${ }^{14}$ Automatisierung ist dabei Teil der Technisierung der Wirtschaft. Sie kann weiter unterschieden werden zunächst in Mechanisierung, d.h. der Unterstützung menschlicher Arbeit durch Maschinen (Arbeitsschritt wird durch menschliche Arbeit erledigt, Maschinen bieten Hilfestellung), und in Maschinisierung, d.h. dem Ersatz menschlicher Arbeit durch M aschinen bzw. die Ausführung bestimmter Arbeitsschritte im Produktionsprozess durch Maschinen.

${ }^{15}$ Beispielhaft sei hier auf den programmierbaren Webstuhl von Joseph-M arie Jacquard aus dem Jahr 1805 verwiesen. Siehe auch die von Charles Babbage entworfene Analytical Engine (1837) und die darauf aufbauenden Entwicklungen mechanischer Rechner, die bis in die 1970er Jahre verwendet wurden.

${ }^{16}$ Bereits in den 1930er Jahren entwickelte Konrad Zuse einen frei programmierbaren mechanischen Rechner, der Gleitkommaoperation ausführen sollte (die Z1); dieser war aber aufgrund seiner mechanischen Konstruktion nicht funktionsfähig. Die erste programmgesteuerte binäre Rechenmaschine war im Jahr 1941 dann die Zuse Z3, die Relais-gesteuert operierte. In der Nachkriegszeit wurden in den USA die ersten digitalen Rechner entwickelt.

${ }^{17}$ Zur gleichen Zeit beginnt die Epoche der künstlichen Intelligenz. Der Begriff wird erstmals beim Dartmouth Summer Research Project on Artificial Intelligence im Jahr 1956 verwendet.

${ }^{18}$ Der TM S1000 war ein 4-Bit Prozessor mit 8.000 Transistoren. Vereinfachend gesprochen kann ein 4-Bit-Prozessor vier Bit (binäre Zustände) gleichzeitig während eines Taktes verarbeiten.
} 
Störanfälligkeit verbunden mit geringerem Stromverbrauch möglich. Bereits 1972 wurde von Intel der erste 8-Bit Prozessor vorgestellt (Intel 8008), der doppelt so leistungsstark war. Der Mikroprozessor erlaubte die Entwicklung von Heim- und Arbeitsplatzcomputern und schaffte damit die Grundlage für den weltweiten Einzug der Computertechnik in alle Lebensbereichen in den folgenden Jahrzehnten.

M it der Verbreitung des Internets seit den 1990er Jahren und der seit M itte des ersten Jahrzehnts der 2000er Jahre zunehmenden M obilität digitaler Technik (u.a. durch Smartphones und Cloudcomputing) hat sich die Entwicklung der Computer- und Digitaltechnik durch ihre Weiterentwicklung zur Überallverfügbarkeit (ubiquous technology) noch einmal rasant beschleunigt. Insbesondere die Nutzung und Integration der Digitalisierung in anderen, vormals analogen Technologiebereichen führt zu einer immer weiteren Durchdringung (und damit Abhängigkeit) der Gesellschaft von digitalen Techniken. Selbststeuernde Anlagen, Roboter und vollautomatisierte und vernetzte Fabriken (Smart Factories ${ }^{19}$; Industrie $4.0^{20}$ ) haben Einzug in die weltweite Produktion von Gütern erlangt; intelligente Software und Algorithmen bekommen zunehmende Bedeutung für die Digitalisierung und Automatisierung von Geschäftsprozessen; auch in der Medizin, Pflege und Bildung erweitert sich das digitale Angebot ständig. ${ }^{21}$ Dieser breite Einsatzbereich von Computern und Digitalisierung befördert die Entwicklung sogenannter General Purpose Technologies. Diese Technologien zeichnen sich durch ein großes Anwendungsfeld, ihr bedeutendes Potenzial für Verbesserungen sowie die leichte Kombinierbarkeit mit anderen Technologien aus (Bresnahan und Trajtenberg 1995). Ein prominentes Beispiel einer General Purpose Technology ist das Internet, das sich von einem spezifischen Kommunikationsinstrument zwischen zunächst menschlichen Akteuren zu einem allgemeinen, enträumlichten Kommunikationsmittel zwischen allen Formen von Sendern und Empfängern weiterentwickelt hat, einschließlich der Kommunikation zwischen M aschinen (genannt Internet der Dinge bzw. internet of things).

\subsection{Ein Vergleich mit anderen Formen technischen Fortschritts}

Der Beginn der industriellen Revolution, die zu umfassenden gesellschaftlichen und technischen Veränderungen geführt hat, wird im Allgemeinen auf die Zeit der Einführung der Dampfmaschine im Jahr 1769 datiert. M it ihrer Entwicklung wurde die Abhängigkeit von menschlicher bzw. tierischer M uskelkraft sowie - in der Regel räumlich oder anders begrenzter - natürlicher Kraftquellen (Wasser, Luft)

\footnotetext{
${ }^{19}$ Smart Factory beschreibt die Vision einer selbstorganisierten Produktionsumgebung, in der in Fertigung und Logistik keine menschlichen Eingriffe durch die Verwendung sogenannter cyber-physischer Systeme erforderlich sind.

${ }^{20}$ Industrie 4.0 beschreibt die Verzahnung industrieller Produktion durch Informations- und Kommunikationstechnologie. Auf der Grundlage intelligenter und digital vernetzter Systeme soll eine weitgehend selbstorganisierte Produktion ermöglicht werden, in der menschliche und nicht-menschliche Akteure aller Wertschöpfungsstufen miteinander kommunizieren und kooperieren.

${ }^{21}$ Wesentliche Treiber der Digitalisierung sind nach Mikfeld (2017) die Entwicklung von Informations- und Kommunikationstechnologie, die Sensorik und Robotik, die Vernetzung von Menschen und Dingen sowie die Künstliche Intelligenz und Big Data. Charakteristisch für die Digitalisierung ist eine Flexibilisierung, Autonomisierung und Individualisierung bereits bestehender Prozesse.
} 
überwunden und nutzbare Energie konnte nach Belieben - und vor allem auch mobil - bereitgestellt werden. Dies ermöglichte die Ablösung der M anufakturen und Faktoreien durch Fabriken, M assenproduktion wurde möglich. M it der Einführung von Eisenbahn und Dampfschiff zu Beginn des 19. Jahrhunderts begann zudem das Zeitalter des M assentransports.

Eine einheitliche Definition im Hinblick auf die Anzahl der industriellen Revolutionen gibt es nicht. Robert Gordon (2016) differenziert drei industrielle Revolutionen seit Einführung der Dampfmaschine, um das massive Wirtschaftswachstum der vergangenen 200 Jahre zu erklären. Die erste industrielle Revolution fand dabei zwischen 1770 und 1840 statt und wirkte bis etwa um das Jahr 1900 fort. Die wesentlichen Entwicklungen dieser ersten industriellen Revolution waren die Dampfmaschine, Eisenbahnen und Dampfschiffe, aber auch Webmaschinen und der Übergang von Holz zu Stahl. Die zweite industrielle Revolution prägte die Jahre 1870 bis 1920 und dauerte in ihren Wirkungen bis etwa 1970 fort. Sie ist gekennzeichnet durch Erfindungen und Entwicklungen in allen Lebensbereichen, z.B. durch Elektrizität, Licht, M aschinen, Klimaanlagen; Verbrennungsmotoren, Fahrzeuge und Flugzeuge; Telefon, Radio, Fernsehen; fließendes Wasser, Kläranlage, Verringerung der Kindersterblichkeit durch verbesserte Hygiene; Chemikalien, Kunststoffe, Antibiotika und die moderne M edizin; Veränderungen der Arbeitsbedingungen durch starke Verstädterung. Sie beschreibt damit zugleich den Übergang von der Agrar- zur Industrie- und Dienstleistungsgesellschaft. Als dritte industrielle Revolution bezeichnet Gordon die Unterhaltungs- und Informationstechnik-Revolution. Sie begann in den 1960er Jahren mit der Einführung des Farbfernsehens bis hin zur heutigen Überallverfügbarkeit von Entertainmentangeboten durch Streaming und Timeshifting. Nach der Einführung von M ainframe-Computern folgte die Verbreitung der Personalcomputer (s.o.) und heute das Internet sowie der E-Commerce. Gleichfalls kennzeichnen Veränderungen der Kommunikation durch M obiltelefone und Smart Phones die dritte industrielle Revolution. Die Verbesserung der Produktivität entsteht dabei z.B. durch die Einführung von Geldautomaten, Barcode-Scannern oder den bargeldlosen Zahlungsverkehr (Kreditkarten, EC-Karten).

Die jüngste Entwicklung der Digitalisierung wird in den M edien aktuell auch als vierte industrielle Revolution bezeichnet. Sie ist gekennzeichnet durch die Digitalisierung der Produktion auf der Grundlage mehrerer General Purpose Technologies und verbunden mit der weit verbreiteten Überzeugung, dass sie Wirtschaft und Gesellschaft tiefgreifend verändern wird (Kurz 2017). Pointierter formulieren es Brynjolfsson und M cAfee (2015): In Analogie zur industriellen Entwicklung nach Einführung der Dampfmaschine im Jahr 1769 und dem dadurch ausgelösten wirtschaftlichen Wachstum sprechen sie vom Beginn des zweiten M aschinenzeitalters durch die Computerisierung und Digitalisierung, d.h. der zweiten Industrialisierung. ${ }^{22}$

\footnotetext{
${ }^{22}$ So der Titel ihres Buches: The Second Machine Age, Brynjolfsson und M cAfee (2015).
} 
Brynjolfsson und McAfee begründen diese Definition dabei über das (mögliche) exponentielle Wachstum der Wirtschaft durch die Digitalisierung. Seit der Industrialisierung hat die gesellschaftliche und technische Entwicklung zwar exponentiell zugenommen, allerdings geschah dies vergleichsweise langsam. Die Dampfmaschinen waren zunächst sehr ineffizient: sie schöpften nur rund ein Prozent der von der verbrannten Kohle freigesetzten Energie ab. Watts Weiterentwicklungen zwischen 1765 und 1776 steigerten diesen Wert um das Dreifache. Obwohl die Dampfrevolution mehrere Jahrzehnte benötigte, um sich zu entfalten, war es dennoch die größte und schnellste Transformation der Weltgeschichte. Die Weiterentwicklungen und neuen technischen Erfindungen erreichten in der Folge über die mehr als 200 Jahre deutlich höhere Wirkungsgrade ${ }^{23}$; im Vergleich zur Dampfmaschine von 1776 hat sich dieser Wirkungsgrad aber erst rund vier $\mathrm{M}$ al exponentiell verdoppelt. Die technische Entwicklung vollzieht sich daher bisher über einen recht langen Zeitraum.

Die Verdopplung der Prozessorleistung, wie oben für die Jahre 1971 und 1972 erläutert, ist demgegenüber paradigmatisch für die Entwicklungen in der Computertechnik insgesamt. Bereits im Jahr 1965 hat Gordon Moore (M itbegründer von Intel) die Feststellung abgegeben, die als Moore's Law weite Bekanntheit erreicht hat: „Die Komplexität hat sich mit minimalen Komponentenkosten etwa um den Faktor zwei pro Jahr erhöht. [...] Das dürfte sich zumindest kurzfristig fortsetzen, wenn nicht gar steigern. Auf längere Sicht ist die Zuwachsrate nicht ganz so gewiss, obschon es keinen Grund zu der Annahme gibt, dass sie nicht mindestens zehn Jahre lang mehr oder minder konstant bleibt." (zitiert nach Brynjolfsson und M cAfee, 2015, S. 54f.). Tatsächlich hat sich diese Einschätzung als viel zu konservativ herausgestellt, da die Entwicklung viel länger anhielt. Auch wenn für die Verdopplungsfrist der allgemeinen Rechnerleistung heute etwa 18 M onate angesetzt werden, gilt M oore's Law bereits seit mehr als einem halben Jahrhundert. ${ }^{24}$

Der technische Fortschritt der digitalen Ära folgt gemäß der empirischen Beobachtung konsistent einem exponentiellen Pfad. Er schreitet damit viel rasanter voran als alle Entwicklungen bisher. ${ }^{25}$ Durch die entstehenden Preis-, Gewichts- und Leistungsfortschritte wird der Einsatz immer vielfältigerer, aufwändiger Systeme möglich, die ständig weitere Lebensbereiche erfassen. So hat die Digitalisierung zu einer grundlegenden Umwälzung geführt, in der viele bisher analoge Sensoren digitalisiert worden sind (M ikrofone, Kameras, Beschleunigungsmesser etc.). Gerade die Erfassung und Abbildung der Umwelt

\footnotetext{
${ }^{23}$ Heutige Dieselmotoren für Automobile erreichen einen Wirkungsgrad von rund 35 Prozent, große Schiffsdieselmotoren Wirkungsgrade von rund 50 Prozent.

${ }^{24}$ M oores Gesetz ist im Eigentlichen kein Gesetz, sondern nur eine Feststellung aus der Beobachtung der Entwicklung der Computerbranche.

${ }^{25}$ Brynjolfsson und M CAfee (2015) geben hierzu ein anschauliches Beispiel: Im Jahr 1996 war der ASCI Red der schnellste Supercomputer der Welt; er erreichte 1997 eine Rechenleistung von 1,8 Teraflop (eine Billion Fließkommaoperationen pro Sekunde) bei einem Verbrauch von $800 \mathrm{~kW} / \mathrm{h}$ und Kosten von rund $55 \mathrm{M} \mathrm{io}$. US-Dollar. 2005 hatte die Sony PlayStation 3 die gleiche Rechenleistung bei 200W/h Verbrauch und Kosten von 500 USDollar.
} 
(insb. in der Robotik) und die entsprechende Adaption führen zu immer höherer Autonomie digitaler M aschinen (environmental control; Autor (2015)). Verstärkt werden diese Effekte durch zugleich fortschreitende Entwicklungen im machine learning, d.h. der Verbesserung der Trefferwahrscheinlichkeiten aufgrund einer immer größeren Informationsfülle und schnellerer Algorithmen (Autor, 2015). Hierbei spielen auch Netzwerkeffekte eine besondere Rolle: der Wert digitaler Ressourcen steigt durch zusätzliche Nutzer für alle Nutzer. ${ }^{26}$ Die allumfassende Digitalisierung beschleunigt den technischen Fortschritt daher weitergehend. Neben den Wirkungen auf Produktion von Waren und Dienstleistungen führt dies auch zu fundamentalen Änderungen der gesellschaftlichen und politischen Prozesse, z.B. durch die sozialen Netzwerke (vgl. hierzu Petersen (2015)).

Brynjolfsson und M cAfee (2015) stellen vor dem Hintergrund dieser Entwicklungen die These auf, dass Computer und andere digitale Errungenschaften die gleiche Wirkung auf unsere geistigen Fähigkeiten haben, wie die Dampfmaschine (und ihre Ableger) sie auf die M uskelkraft hatte und hat. Frühere Einschränkungen werden damit überwunden, da die Geisteskraft für Fortschritt und Entwicklung mindestens ebenso wichtig ist wie die physische Kraft. Das zweite M aschinenzeitalter soll der Menschheit durch diesen kräftigen und ungekannten Impuls auf die Geisteskraft daher einen enormen Schub verleihen (genauso wie ihr physisches Pendant in der ersten industriellen Revolution).

Kritischer wird dies hingegen von Gordon (2016) gesehen, der geringere Erwartungen an die Wirkungen der technischen Revolution durch Computerisierung und Digitalisierung hat. Bereits vor gut zwanzig Jahren illustrierte er den abnehmenden Grenznutzen der Computerisierung und Digitalisierung am Beispiel der Einführung von Textverarbeitungsprogrammen (Gordon 2000): Der Einbau eines Speichers in eine Schreibmaschine verringerte dabei die erforderlichen Kapazitäten für wiederholtes Schreiben zunächst erheblich. Die Zuwächse in Prozessorgeschwindigkeit und Speicherkapazität haben in der Folge aber nicht zu gleichen Vorteilen in der Produktivität bei der Erstellung von M anuskripten geführt. Die Einführung der graphischen Oberfläche mag hierzu noch einen Beitrag geleistet haben, spätere Weiterentwicklungen haben aber einen Grenznutzen von mehr oder weniger Null. Gordon (2000) will mit diesem Beispiel deutlich machen, dass die wirkliche Revolution durch die Computertechnik bereits zu Beginn erfolgte (d.h. am Beispiel: das Ende des wiederholten Schreibens und die M öglichkeiten zur Umarbeitung von Texten, während der übrige Text von selbst formatiert wird). Die weiteren Entwicklungen brachten keine vergleichbar großen Fortschritte. Allgemeiner formuliert er:

"The productivity enhancement of WYSIWYG [What You See Is What You Get] was minor in comparison, and what was contributed by the final step to the latest version of Word for W indows, beyond some ease of training for novice users, escapes me. As the computer industry has

\footnotetext{
${ }^{26}$ Google zeigt auf seiner Suchseite z.B. detaillierte Informationen über Öffnungszeiten, Stoßzeiten und durchschnittliche Verweildauern von Kunden an; diese werden aus den Bewegungsprofilen von Smartphonebesitzern abgeleitet. Ähnliche Algorithmen werden auch in der Verkehrsnavigation, insb. um Staus zu entdecken und Umfahrungen zu berechnen, eingesetzt.
} 
developed, the steady decline in the prices of computer characteristics has fueled the development of increasingly complex software with high requirements for speed and memory required by graphical point-and-click interfaces that yield increasingly small increments of true functionality. The race between hardware capability and software requirements has been aptly summed up in the phrase, "What Intel giveth, Microsoft taketh away."' (Gordon 2000, S. 63)

Aus diesen Zeilen wird deutlich, dass nicht allein die Möglichkeiten (siehe Abschnitt 2) für neue Kombinationen entscheidend sind, sondern auch ihre Durchsetzung am Markt. Konkreter am Beispiel der Computerisierung und Digitalisierung heißt das, dass die Verdoppelung der Prozessorleistung (bzw. anderer technisch relevanter Größen) nicht zwingend mit einer Erhöhung oder gar Verdopplung des Nutzens einhergeht. Interpretiert im Sinne Schumpeters kann dies bedeuten, dass die technischen Neuerungen der Gegenwart differenzierter zu betrachten sind. So können einige (z.B. die Einführung der Textverarbeitung) als wirkliche Innovationen das Gleichgewicht verschieben; andere müssen aber vielmehr als zu erwartende trendgemäße Weiterentwicklungen verstanden werden, deren Potenzial zur Produktivitätsverbesserung deutlich beschränkter ist - und gegebenenfalls die zusätzlichen Investitionen nicht rechtfertigt.

Ein Aspekt von Computerisierung und Digitalisierung, der als grundsätzliche Innovation gelten kann, ist die Verringerung der Grenzkosten von repetitiven Arbeitsgängen bei gleichbleibender Qualität und gleichzeitiger Verringerung der Transportkosten. Baldwin (2016) diskutiert die aktuellen Entwicklungen und Perspektiven durch die Informations- und Kommunikationstechnik in einer Analyse der Schlüsselparameter für die vorangegangenen Globalisierungswellen. Allen Globalisierungswellen ist dabei gemeinsam, dass sie den Unternehmen neue Produktions- und Absatzmöglichkeiten (siehe Schumpeter) erschlossen haben. In der industriellen Revolution haben sich die Transportkosten durch die Entwicklung der Dampfmaschine und den damit verbundenen Entwicklungen von Dampfschiff (zunächst mit Holzrümpfen und ab den 1870er Jahren dann aus Stahl mit noch größeren Lade- und Bunkerkapazitäten) und Eisenbahn deutlich verringert. In der Zwischenkriegszeit der Weltkriege im 20. Jahrhundert wurden die globalen Handelsaktivitäten durch höhere Zölle und eine stärkere Artikulation der Nationalstaaten wieder temporär geringer, bevor in der Nachkriegszeit die Ära der Globalisierung (begleitet durch Freihandelsabkommen) an Bedeutung weiter gewann. Hierbei spielten weitere technische Neuerungen - u.a. Dieselmotor und Container - eine besondere Rolle, um die Transportkosten im Handel noch weiter zu senken.

Im Gegensatz dazu hat die aktuelle Globalisierung auf Grundlage der Informations- und Kommunikationstechnologie eine andere Qualität: sie basiert vielmehr auf dem immer günstiger werdenden Transport von Know-How, der eine Verlagerung der Produktion an den immer günstigsten Ort ermöglicht (hat). Allerdings war die erste Phase der durch die Informations- und Kommunikationstechnologie eingeleiteten bzw. ermöglichten Globalisierung auf die Produktion beschränkt. Die Dienstleistungen - 
auch die mit Wartung und Beratung verbundenen - waren nicht exportierbar und blieben von der Globalisierung weitgehend unberührt.

Durch das Internet und die immer größeren übertragbaren Datenmengen ist hier aber diese letzte Barriere gefallen: Dienstleistungen können global eingesetzt und erbracht werden und reduzieren die lokal erforderliche Arbeit (z.B. in der Wartung von M aschinen oder der Aktualisierung) drastisch. Dies führt zu einer virtuellen M igration der Arbeit, die die Grenzen der Nationalstaaten nicht mehr kennt. Hiermit verbunden sind vielfältige gesellschaftliche und politische Folgen. Wenn Schlüsselfaktoren, die den komparativen Vorteil eines Landes ausmachen relativ transaktionskostenfrei Landesgrenzen überschreiten können, spielt die M obilität der einzelnen Faktoren für die Entwicklung und Verfolgung wirtschaftspolitischer Ziele eine zentrale Rolle. Baldwin zeigt damit im Vergleich zu Brynjolfsson und M cAfee oder Gordon einen entscheidenden Punkt der Digitalisierung auf, ohne zu optimistisch bzw. zu pessimistisch bezüglich des tatsächlichen Potenzials zu sein.

\section{Wirkungen der Computerisierung und Digitalisierung}

\subsection{Die Suche nach den Produktivitätseffekten: Das Solow Computer Paradoxon}

Doch welche ökonomischen Wirkungen haben die Computerisierung und Digitalisierung? Geradezu paradigmatisch für die Schwierigkeiten, den technischen Fortschritt der Computer (zunächst bis in die 1990er Jahre) zu erkennen, steht das sogenannte Solow Computer Paradoxon. Solow (1987, S. 36) stellte fest: „You can see the computer age everywhere but in the productivity statistics." Grundlage dieser Feststellung war die Verringerung der Produktivität der US-amerikanischen Wirtschaft in den 1970er und 1980er Jahren. ${ }^{27}$ Über den gleichen Zeitraum wurden nicht nur beachtliche Fortschritte in der Zunahme der Rechenleistung von Computern gemacht, auch waren die Investitionen in die Informationstechnik (IT) beträchtlich. Trotzdem verlangsamte sich das Produktivitätswachstum der gesamten US-amerikanischen Wirtschaft. Diese Verlangsamung war zum Teil sogar stärker in Branchen, die besonders in IT investiert hatten. Den M ittelpunkt des Paradoxons stellt entsprechend die Diskrepanz zwischen den Investitionsindikatoren und den Wertschöpfungsindikatoren, vor allem dem Nationalprodukt, dar.

M aßgebliche Kritik an dem Paradoxon begründete sich jedoch rasch darin, dass es als Ergebnis nicht haltbarer Annahmen über den Effekt und die Verbreitung von Technologie zustande gekommen sein könnte. ${ }^{28}$ David (1990) führt hierzu die historischen Parallelen der Einführung von Dampfmaschine 0der Elektrizität an. Die Erträge dieser beiden revolutionären Technologien wurden auch nur langsam,

\footnotetext{
${ }^{27}$ Das Wachstum der Arbeitsproduktivität der USA lag in den 1960er Jahren bei 3\% p.a., in den 1990er Jahren nur noch bei rund $1 \%$ p.a.

${ }^{28}$ Vergleiche hierzu auch die vielzitierte Studie von Brynjolfsson (1993). In dieser stellt er fest (ebd., S. 76): „Research on IT and productivity has been disappointing, not only because it has only exacerbated apprehension
} 
nach mehreren Dekaden Verzögerung sichtbar, da sowohl die Verbreitung der Technologien, als auch die Anpassung der Wirtschaft an dieselben diese Zeiträume benötigten. Erst Ende der 1990er Jahre konnten Hinweise auf einen zeitverzögerten IT-bedingten Produktivitätsanstieg gezeigt werden, siehe

\section{z.B. Dewan und Kraemer (1998).}

Unbenommen von dieser Diskussion erwarten Brynjolfsson und M cAfee (2015) entsprechend ihrer Hypothese des zweiten M aschinenzeitalters einen deutlichen Anstieg der Produktivität durch die technologischen Entwicklungen. Ausgehend davon formulieren sie klare (und dramatische) Erwartungen für den US-amerikanischen Arbeitsmarkt: zum einen gehen sie davon aus, dass alle Sektoren - und insbesondere die IT-intensiven Sektoren - deutliche Produktivitätsanstiege verzeichnen müssen, und zweitens, dass durch den Ersatz mit Maschinen die Zahl der Arbeitnehmer abnehmen und die Rolle der Arbeit in der Produktion geringer werden wird.

Die Ergebnisse von Acemoglu et al. (2014) zeigen jedoch, dass diese Erwartungen (zumindest bisher) empirisch nicht nachweisbar sind. Das Solow Computer Paradoxon hat somit auch nach 30 Jahren noch Bestand. Da IT inzwischen in allen Wirtschaftszweigen verwendet wird, betrachten Acemoglu et al. (2014) in ihrer Analyse IT-nutzende Sektoren - im Gegensatz zu den in der früheren Literatur untersuchten IT-produzierenden Sektoren im verarbeitenden Gewerbe. Es wird deutlich, dass das Produktivitätswachstum keine eindeutigen Ergebnisse zeigt, und außerdem vom M aß der IT-Intensität abhängt. Insbesondere nach den späten 1990er Jahren lässt sich kein schnelleres Produktivitätswachstum in den IT-intensiven Industrien mehr feststellen. Wichtiger noch ist das Ergebnis, dass das schnelle Wachstum in IT-intensiven Branchen mit einem sinkenden Output einhergegangen ist, und daher allein durch eine noch stärker gesunkene Beschäftigung in diesen Bereichen erklärt wird. Dies steht im klaren Gegensatz zur Prognose von Brynjolfsson und M cAfee (2015): wenn IT tatsächlich die Produktivität steigern würde und dadurch die Kosten reduzieren könnte, müsste dies in einem gestiegenen bzw. steigenden Output zum Ausdruck kommen, zumindest in den IT-intensiven Bereichen der Wirtschaft.

\subsection{Ergebnisse zum Einfluss des Computers auf Löhne und Beschäftigung}

Die Wirkungen bzw. Effekte der Computerisierung auf die Lohnverteilung und die Lohnungleichheit wurden in den vergangenen rund zweieinhalb Dekaden von vielen Autoren und für viele Länder untersucht. Hierbei sind sowohl die methodischen, als auch die informationellen Entwicklungen über die Zeit beachtlich. Sie haben das Verständnis über die notwendigen Zusammenhänge stetig verbessert.

about the ultimate value of billions of dollars of IT investment, but also because it has raised frustrating concerns with the measures and methods commonly used for productivity assessment. [...] While a number of dimensions of the paradox are disturbing and provoking, we still do not have a definitive answer to the question of whether the productivity impact of IT has actually been unusually low. [...] [T]he underlying data underscores the possibility that measurement difficulties may account for the lion's share of the gap between our expectations for the technology and its apparent performance." 
Während die älteren Studien (in den 1990er Jahren) angebotsorientierte Untersuchungen sind, d.h. im Wesentlichen die Veränderungen der Qualifikationen der Arbeitsanbieter in differenzierter Weise untersuchen, wurden ab den 2000er Jahren zunehmend nachfrageseitige Aspekte in die Analysen aufgenommen.

Eine Zunahme der Lohnungleichheit wurde zunächst für die USA für die 1980er Jahre festgestellt (Katz und M urphy, 1992). Diese betraf insbesondere den oberen Teil der Lohnverteilung. Als maßgeblich für diese Entwicklung wurde der faktorverzerrte technologische Fortschritt gesehen, der die Löhne höher Qualifizierter stärker und schneller steigen ließ als die Löhne geringer Qualifizierter (Acemoglu, 2002). Die aus dieser Beobachtung abgeleitete SBTC-Hypothese schien durch die Entwicklungen der Lohnungleichheit der 1970er und 1980er Jahre nicht verworfen werden zu können (Katz und Autor, 1999). Oder anders ausgedrückt: sie schien die Entwicklungen gut erklären zu können. Seit dieser Zeit hat die Literatur ihren Schwerpunkt verschoben, da sich die Beschäftigung zunehmend an beiden Enden der Lohnverteilung konzentriert hat und dabei die mittleren Beschäftigungen und Löhne deutlich abgenommen haben (siehe z.B. Autor et al. 2003, 2006 und 2008), was mit der SBTC-Hypothese nur bedingt in Einklang zu bringen ist. Für dieses Aushöhlen des Arbeitsmarkts hat sich der Begriff der „Polarisierung" verbreitet.

Der Anstieg der Lohnungleichheit wurde dabei zunächst als angelsächsisches Phänomen wahrgenommen. Krugman (1994) stellte hierzu fest, dass die hohe und anhaltende Arbeitslosigkeit in den europäischen Staaten und die Lohnungleichheit in den angelsächsischen Ländern zwei Seiten derselben M edaille wären. Während in Europa Lohnrigiditäten zu Beschäftigungseffekten bzw. Arbeitslosigkeit führten, haben flexiblere Löhne in den angelsächsischen Ländern mehr Ungleichheit ausgelöst. Seit den 1990er Jahren wurde aber auch eine Zunahme der Lohnungleichheit in Europa bzw. in Deutschland festgestellt (siehe z.B. Kohn, 2006; Gernandt und Pfeiffer, 2007; Dustmann et al., 2009). ${ }^{29}$

Als Erklärungen für die Zunahme der Lohnungleichheit im unteren Teil der Verteilung konnten dabei die zunehmend geringere Tarifvertragsabdeckung sowie der Bedeutungsverlust der Gewerkschaften, insbesondere seit Ende der 1990er Jahre identifiziert werden. Außerdem haben auch die sogenannten Hartz-Reformen durch geringere implizite M indestlöhne (hervorgerufen durch die Absenkung des $\mathrm{Ni}$ veaus der Lohnersatzleistungen) und die Flexibilisierung von Arbeitsverträgen zu Lohnsenkungen und

\footnotetext{
${ }^{29}$ Die Betrachtung der Ungleichheit(-entwicklung) allein in den Industrieländern überdeckt dabei zugleich die eher gegenläufige Entwicklung der Ungleichheit allgemein. M ilanovic (2016) analysiert die jüngere Entwicklung der globalen Ungleichheit und versucht einen Ausblick auf die zu erwartenden Entwicklungen. Hierbei unterscheidet er Entwicklungen der sogenannten globalen Mittelklasse sowie des obersten Perzentils der Gesellschaft. Unterschiede ergeben sich dabei durch Ungleichheiten innerhalb der Länder, aber auch durch Unterschiede zwischen den Ländern. M ilanovic greift zur Erklärung der Ungleichheitsentwicklung in den Ländern von der präindustrialisierten über die industrialisierte bis zur digitalen Phase auf die Kuznet-Wellen bzw. KuznetZyklen als Zusammenspiel ökonomischer und politischer Faktoren zurück.
} 
Beschäftigungsanstiegen im unteren Teil der Lohnverteilung beigetragen (Dustmann et al., 2014). Aufgrund der Besonderheiten der Lohnbildung in Deutschland, die trotz verschiedener Anpassungen im Grundsatz beibehalten wurde, sehen Fitzenberger et al. (2011) die Veränderungen der Lohnungleichheit am unteren Ende der Verteilung aber als episodisch auftretende Ereignisse an, und nicht, wie z.B. Autor und Dorn (2013, s.u.) vermuten, als Ergebnis einer permanenten bzw. verfestigten Arbeitsmarktpolarisierung. Diese Ansicht wird auch durch die Ergebnisse von Myck et al. (2011) gestützt. Sie zerlegen die Lohnungleichheit in einen permanenten und einen vorübergehenden Teil. Ihre Ergebnisse zeigen, dass die permanente Lohnungleichheit ihren Höhepunkt in Deutschland bereits 2001 hatte und seitdem wieder abgenommen hat. Die danach beobachtete Polarisierung der Löhne interpretieren sie entsprechend eher als vorübergehendes Phänomen. Institutionelle Gründe können für den Anstieg der Lohnungleichheit im oberen Teil der Verteilung in Deutschland nicht angeführt werden. Sie hat seit den 1980er Jahren leicht zugenommen; wesentlich dafür waren Änderungen in der Qualifikationsstruktur der Beschäftigten (vgl. Dustmann et al., 2009).

Obwohl die vorgestellten Analysen sehr ausführlich die Entwicklungen der Lohnungleichheit und ihre möglichen Gründe untersuchen, wird der Versuch der Erklärung durch technischen Fortschritt und insbesondere Computerisierung nicht überall unternommen. Eine erste Studie, die die Bedeutung der Computer für den Arbeitsmarkt explizit untersucht, hat Krueger (1993) vorgelegt. Er sucht mögliche Erklärungen für die gestiegene Lohnungleichheit in den USA während der 1980er Jahre, d.h. stärker steigende Bildungsrenditen für College-Absolventen im Vergleich zu High-School-Absolventen und sieht die Auswirkungen der Computerrevolution hier als zentral an. ${ }^{30}$ Seine Ergebnisse zeigen, dass die Computer komplementär zur Arbeit der Hochqualifizierten sind. Beschäftigte, die mit Computern arbeiten, verdienen ceteris paribus zehn bis 15 Prozent höhere Löhne. Da insbesondere die Hochqualifizierten davon profitieren, macht der Computergebrauch nach Krueger (1993) einen erheblichen Teil der Zunahme dieser Rendite aus. Die Ergebnisse der Studie stützen entsprechend eindeutig die SBTCHypothese.

Dass solch große Bildungsrenditen allein durch die Computernutzung erreicht werden, war (und ist) kein unumstrittenes Ergebnis. So stellen z.B. DiNardo und Pischke (1997) das von Krueger (1993) gefundene Lohndifferential fundamental in Frage. Sie zeigen, dass Computer per se nicht die Erklärung sein können. Hierzu replizieren sie zunächst Kruegers Analyse für Deutschland und kommen zu ähnlich großen Lohndifferentialen der Computernutzung. Bei zusätzlicher Berücksichtigung unterschiedlicher Berufe (d.h. den damit verbundenen branchenspezifischen Lohnunterschieden) in der Schätzung zeigt

\footnotetext{
${ }^{30}$ Als Computerrevolution bezeichnet er die enorme Verbreitung der Computertechnik in den 1980er Jahren über alle Firmen hinweg, die mit einem deutlichen Preisverfall der M ikrocomputer verbunden und auch durch diesen bedingt war. Am Ende der 1980er Jahre arbeiteten bereits rund 40 Prozent der Beschäftigten in den USA mit einem Computer.
} 
sich aber bereits eine Halbierung des Lohndifferentials. Anschließend analysieren sie die Lohndifferentiale anderer Arbeitsmittel (sog. „white collar“- Arbeitsmittel, u.a. Taschenrechner, Bleistift, Telefon, sitzende Tätigkeit) und finden ein vergleichbar großes Lohndifferential wie durch die Nutzung der Computer. Dass die Beschäftigten derart große Renditen durch die Verwendung von Bleistiften erreichen können (angesichts der Alphabetisierungsrate von 99\% in Deutschland), macht DiNardo und Pischke (1997) skeptisch gegenüber den von Krueger (1993) ermittelten Lohnprämien der Computer. Die Ergebnisse spiegeln also keine direkten Produktivitätseffekte wider, sondern berichten über Korrelationen. Aus ihren Ergebnissen schließen sie daher, dass vielmehr unbeobachtete Fähigkeiten hierfür von Bedeutung sein müssen. Zwei wesentliche Implikationen ergeben sich:

1) Computer sind nur in Verbindung mit bestimmten Fähigkeiten produktiv und

2) Computer sind nur in bestimmten Berufen wertvoll.

Personen mit diesen bestimmten (und knappen) Fähigkeiten wählen daher die Berufe, in denen ihre Fähigkeiten besonders wertvoll sind (Selbstselektion). Da diese Fähigkeiten in den verwendeten Daten aber nicht beobachtet werden können, sind die hohen geschätzten Renditen möglicherweise ein Trugschluss durch Selektionsverzerrung. Für DiNardo und Pischke (1997) bleibt daher die Frage, ob Computer zu einer Produktivitätssteigerung führen, ungelöst.

Beide Studien sind dabei exemplarisch für eine ganze Reihe von Artikeln in den führenden wirtschaftswissenschaftlichen Zeitschriften um die Jahrtausendwende zu sehen, die der Frage der Bedeutung der Computer auf den Arbeitsmarkt nachgehen. Die zentrale Schwierigkeit einer eindeutigen Beurteilung ergibt sich aus dem Problem, die Rolle der Computer im Arbeitsprozess zu verstehen und zu quantifizieren. Dies führt zu den ambivalenten Ergebnissen. Wulff Pabilonia und Zoghi (2005) fassen die Überlegungen der Debatte um die Jahrtausendwende in etwa wie folgt zusammen: wenn Computer die Grenzproduktivität der Arbeit erhöhen, dann sollten Beschäftigte einen Lohnaufschlag durch die Computernutzung realisieren können. Dieser Produktivitätsvorteil könnte dadurch entstehen, dass Unternehmen sich wiederholende Aufgaben automatisieren (könnten) und sich dadurch die Kapazitäten der Beschäftigten für Aufgaben wie Problemlösung, Innovation und zwischenmenschliche Interaktion erhöhen (würden). Dies könnte dann eine Verschiebung der Gesamtproduktivität der Beschäftigten erklären. Um diesen Punkt zu unterlegen, lösen sie in ihrer eigenen empirischen Untersuchung auf Grundlage kanadischer Daten das Selbstselektionsproblem unter Verwendung von Instrumentvariab- 
len; die so bereinigten Schätzungen machen deutlich, dass die zunächst im einfachen M odell bestätigten hohen Renditen der Computernutzung (ca. 17 Prozent) verschwinden. ${ }^{31}$ Ihre vertiefenden Schätzungen machen zugleich deutlich, dass das Lohndifferential fast vollständig auf die Anwendung von Computerkenntnissen zurückzuführen ist, nicht auf die Nutzung eines Computers per se. ${ }^{32}$

So ambivalent das Bild bezüglich der Bedeutung des Computers für Löhne und Beschäftigung in diesen exemplarischen Studien ist, so vielschichtig sind auch die Diskussionen um die Erklärungsansätze für die Veränderungen der Lohnungleichheit seit den 1980er Jahren. In ihrer bedeutenden Studie zu den Trends in der Lohnungleichheit in den USA seit den 1980er Jahren fassen Autor et al. (2008) die zwei zentralen Schlussfolgerungen der Diskussion zusammen. Auf der einen Seite sind Verschiebungen im Angebot und der Nachfrage nach Qualifikationen (Höherqualifizierung) verantwortlich. Diese haben im Zusammenspiel mit dem Bedeutungsverlust von Gewerkschaften und der realen Verringerung des M indestlohns (d.h. von Institutionen zum Schutz gering- und mittelqualifizierter Beschäftigter) zum Anstieg der Ungleichheit geführt. Befördert wurde diese Entwicklung dabei zusätzlich durch die Computerrevolution, die die Nachfrage nach höheren Qualifikationen überproportional steigen ließ, während das Angebot an Hochqualifizierten relativ dazu langsamer wuchs. Demgegenüber stehen auf der anderen Seite die von Autor et al. (2008) (M IT) als „Revisionisten“ bezeichneten Autoren, insb. John DiNardo (M ichigan), David Card (Berkeley) oder Thomas Lemieux (Vancouver). Sie argumentier(t)en, dass die Lohnungleichheit maßgeblich durch nicht-M arktfaktoren gestiegen ist und dass die Entwicklung nur episodischen Charakter hat (d.h. nicht länger anhaltend fortschreiten sollte). ${ }^{33}$

Für Autor et al. (2003) ist das Wachstum der Informations- und Kommunikationstechnologie (IKT) aber der zentrale Auslöser für die Polarisierung (und damit die Zunahme der Ungleichheit) des Arbeitsmarkts. Als Polarisierung wird die gleichzeitige Zunahme der Beschäftigung am oberen und unteren Ende der Verteilung zu Lasten der Beschäftigungen mit mittleren Einkommen verstanden. Insbesondere die durch die IKT bedingte rapide und deutliche Kostenreduzierung, die durch den immer besseren und schnelleren Ersatz leicht kodifizierbarer Tätigkeiten im Arbeitsprozess durch Computer er-

\footnotetext{
${ }^{31}$ Diese naiven Renditen der Computernutzung haben dabei eine vergleichbare Größenordnung wie in Studien für andere entwickelte Länder zuvor.

32 Obwohl die Studien zeigen, dass Computerkenntnisse eine wesentliche Rolle für den Einkommensunterschied spielen, impliziert dies nicht zugleich das Erfordernis, diese in expliziter Form bereits vor Arbeitsmarkteintritt zu vermitteln. Borghans und ter Weel (2004) untersuchen hierzu, ob eine solche Vermittlung gleichbedeutend mit der Vermittlung von mathematischen oder orthographischen Kenntnissen erfolgen sollte. Ihre Ergebnisse zeigen, dass dies zumindest für die 1990er Jahre nicht empfehlenswert ist. Abgesehen von der kleinen Gruppe von Programmierern, sind die erforderlichen Fähigkeiten zur Computernutzung meist leicht während der Arbeit erlernbar und binden auch nur geringe Investitionen. In der Wertschöpfung zählt die Bedienung eines Computers zu den Routineaufgaben der Beschäftigten. Daher sind keine besonderen Lohnaufschläge für diese Tätigkeit zu erwarten, die eine besondere Investition in das Humankapital rechtfertigen würden.

33 Siehe hierzu u.a. Card und DiNardo (2002) oder Lemieux (2006).
} 
reicht wurde, hat die Arbeitgeber zur Substitution von menschlichen Tätigkeiten durch (digitale) Technologie veranlasst. Leicht kodifizierbare Aufgaben (oder Routinetätigkeiten), ob manuell oder kognitiv, sind dabei charakteristisch für Beschäftigte mit mittleren Einkommen und mittleren Qualifikationen.

\subsection{Der Tätigkeiten-basierte Ansatz zur Abbildung von Beschäftigungs- und Lohneffek- ten der Automatisierung}

Um die Substitutionspotenziale von M enschen durch Computer sichtbar zu machen, schlagen Autor et al. (2003) ein Modell vor, dass nicht die Fähigkeiten, sondern die mit diesen Fähigkeiten ausgeführten Tätigkeiten in den Mittelpunkt stellt. Dieses Modell wurde von vielen Autoren mit Daten unterschiedlicher Länder empirisch getestet und hat bereits eine beachtliche Verbreitung erhalten. Während das Lehrbuchmodell (oder kanonische Modell) die Produktionsfunktion vom Menschen ausgehend definiert (M ensch und Kapital wirken in einer bestimmten arbeitsteiligen Beziehung zusammen in der Erstellung eines Produkts), weist es Arbeit und Kapital in ausgeprägter Form statische Rollen zu. Die Eigenschaften, die diese Rollen unverwechselbar machen, sind dabei nicht abschließend oder klar definiert. Auch die Interaktion beider Faktoren beschränkt sich in der Regel auf Substitution oder Komplementarität. Dementsprechend sind in diesem M odell die Identität eines Faktors und seine Rolle in der Produktionsfunktion gleichbedeutend, während die Grenzen in der Wirklichkeit viel durchlässiger sind (Autor, 2013).

Der technologische Fortschritt und insbesondere die Digitalisierung ermöglichen, dass Maschinen die menschliche Arbeit in bestimmten Tätigkeiten ersetzen, während sie sie in anderen Tätigkeiten ergänzen. Den Hauptgrund dafür sieht Autor (2013) im Entwicklungsprozess der Arbeitsteilung. Neue bzw. neuartige Aufgaben werden zunächst von Beschäftigten durchgeführt, da diese flexibel und anpassungsfähig sind. Sie können auch Anweisungen ausführen, denen die erforderliche Exaktheit für eine automatische Durchführung (noch) fehlt. Später im Arbeitsprozess werden diese Aufgaben dann formalisiert und kodifiziert, um sie automatisiert auszuführen und die entsprechenden Kostenvorteile zu realisieren. Die Verlagerung der Tätigkeiten von Aufgaben menschlicher Arbeit (labor tasks) zu Aufgaben maschineller Erfüllung (capital tasks) spielt die Schlüsselrolle in der veränderten Struktur der Arbeitsnachfrage in den Industrieländern. Im Grundsatz findet sich eine vergleichbare Überlegung der Arbeitsteilung bereits im berühmten Stecknadelbeispiel von Adam Smith, in dem Smith die Automatisierung als Teil der Arbeitsteilung beschreibt.

Das formale M odell hierzu ist ausführlich in Acemoglu und Autor (2011) beschrieben und verallgemeinert das M odell von Acemoglu und Zilibotti (2001). Es erlaubt in flexibler und handhabbarer Weise die Interaktion zwischen Fertigkeiten, technologischen Fähigkeiten, potenziellen Handels- und Offshoring-M öglichkeiten zu analysieren und dadurch die Wirkungen auf die Nachfrage nach Produktionsfaktoren, die Zuordnung von Faktoren zu Aufgaben im Wertschöpfungsprozess und letztlich ihren Preis 
bzw. Lohn zu bestimmen. Anders als in der traditionellen Unterscheidung verschiedener Produktionsfaktoren bilden allein Aufgaben (bzw. Tätigkeiten; tasks), die kombiniert werden müssen, um Waren und Dienstleistungen zu erzeugen, die Basis des M odells. Hierdurch überwinden Acemoglu und Autor (2011) die übliche (und artifizielle sowie einschränkende) Unterscheidung zwischen Arbeit, Kapital, Handel und technischem Fortschritt.

Eine Tätigkeit ist dabei eine Arbeitseinheit, die Output erzeugt. Diese muss nicht durch menschliche Arbeit erbracht werden. Die Fertigkeiten (skills) der Beschäftigten beschreiben daneben den Bestand an Fähigkeiten, der für die Ausführung verschiedener Tätigkeiten genutzt werden kann. Die Beschäftigten nutzen also ihre Fertigkeiten bzw. Fähigkeiten, um Tätigkeiten zu vollziehen, und bekommen hierfür im Austausch ihren Lohn. Im Tätigkeiten-basierten Ansatz ist dabei entscheidend, dass Fähigkeiten zur Durchführung der Tätigkeiten zugewiesen werden, um Output zu erzeugen. Es gibt aber keine Äquivalenz zwischen den Fähigkeiten bzw. Fertigkeiten der Beschäftigten (skills) und den Aufgaben (tasks); Fertigkeiten allein produzieren dementsprechend auch keinen direkten Output (Autor, 2013).

Die Grenze zwischen menschlichen (labor tasks) und maschinellen Aufgaben (capital tasks) ist dabei fließend, da die Arbeitsteilung im Produktionsprozess durch (mindestens) zwei Kräfte bestimmt wird. Die erste bezeichnen Acemoglu und Autor (2011) als technologische Macht (technological force); die zweite ist die ökonomische (economic force). ${ }^{34}$ Die technische Entscheidung bzw. M öglichkeit der Substitution wurde bereits oben am Beispiel erläutert: der technische Innovationsprozess geht in der Regel zunächst über den M enschen, da dieser in der Lage ist, neue Aufgaben flexibel und anpassungsfähig auszuführen. Um die Aufgaben zu automatisieren, d.h. menschliche durch maschinelle Arbeit zu ersetzen, müssen die Aufgaben außerdem weit besser verstanden werden, als für ihre einfache Ausführung erforderlich ist. Sie müssen so kodifiziert werden, dass relativ unflexible Maschinen sie halbautonom ausführen können.

Hier schließt die ökonomische Entscheidung an: selbst wenn die Aufgabe vollkommen automatisierbar ist, wird sie jedoch solange nicht automatisiert durchgeführt, solange die Kosten menschlicher Arbeit günstiger sind. Die Überschneidung von technischer Machbarkeit und ökonomischen Kosten (einschließlich der Opportunitätskosten) bedingt dann die tatsächliche Ausgestaltung des Produktionsprozesses. Sie folgt dem Prinzip der komparativen Vorteile, d.h. der Produktionsfaktor mit den geringsten Kosten der Durchführung einer bestimmten Aufgabe wird für die Durchführung gewählt.

\footnotetext{
${ }^{34}$ Diese Unterteilung erinnert an die Formulierung der "neuen Kombinationen“ durch Schumpeter (siehe Abschnitt 2). Sie sind nicht allein durch eine technische Veränderung gegeben, sondern müssen sich auch durchsetzen bzw. durch den Unternehmer durchgesetzt werden. Für die erforderlichen Mittel hat er dazu die Kapitalisten, d.h. Geldgeber, zu überzeugen.
} 
Gegeben die Preise für die verschiedenen erforderlichen Aufgaben sowie die Löhne für die verschiedenen Qualitäten von Beschäftigten (bzw. ihre Fähigkeiten) wählen die Firmen (aber auch die Beschäftigten) die optimale Allokation von (menschlichen und maschinellen) Fähigkeiten zur Ausführung der Aufgaben aus. Der technische Wandel im Modell von Acemoglu und Autor (2011) kann daher die Produktivität von Arbeitnehmern bei allen ihre Aufgaben verändern (wie in den Modellen zuvor). Er kann aber auch nur einzelne Aufgaben betreffen, was dann ihre komparativen Vorteile und damit ihre Einsatzmöglichkeiten im Arbeitsmarkt verändert. Neue Technologien können darüber hinaus die Beschäftigten bei der Erfüllung bestimmter Aufgaben vollständig ersetzen. Alle vier betrachteten Größen (Fähigkeiten der Beschäftigten, technische Leistungsfähigkeit des Kapitals, internationaler Handel und Offshoring, d.h. Verlagerung der Produktion ins Ausland) sind konkurrierende Inputs für die Bearbeitung der erforderlichen Aufgaben. Durch diesen Aufbau, der auf Überlegungen von Kosten und komparativen Vorteilen beruht, kann das Modell die wesentlichen Aspekte der Wertschöpfung in der globalisierten und digitalisierten Welt integrieren und Änderungen der Arbeitsnachfrage bzw. des Arbeitsmarktgleichgewichts modellieren.

\subsection{Neuere Ergebnisse: Polarisierung des Arbeitsmarkts}

Autor et al. (2003) untersuchen die Veränderung der nachgefragten Fähigkeiten am Arbeitsmarkt aufgrund der technologischen Veränderungen von 1960 bis 1998 unter Verwendung des Tätigkeitenbasierten Ansatzes. Ihr wesentliches Argument ist, dass Computer in Abhängigkeit der Ausgestaltung der Arbeitsaufgaben zwei unterschiedliche Dinge tun: auf der einen Seite ersetzen sie die Beschäftigten bei kognitiven und manuellen Aufgaben, die durch klar kodifizierbare Regeln beschrieben werden können. Auf der anderen Seite stellen sie Komplemente für Beschäftigte bei nicht-routinierten, problemlösenden Aufgaben und in komplexen interaktiven bzw. kommunikativen Situationen dar.

Da in der Regel beide Arten von Aufgaben in den einzelnen beruflichen Tätigkeiten vorkommen (d.h. Berufsbilder aus verschiedenen Einzelaufgaben zusammengesetzt sind) und auch nicht die eine Art von (Teil-)Aufgaben vollständig durch die andere ersetzt werden kann, zeigen Autor et al. (2003), dass der technologische Fortschritt durch die Computer tatsächlich zu Veränderungen in der Struktur der beruflichen Aufgaben in verschiedenen Berufen, Branchen und Qualifikationsgruppen geführt hat: die Computerisierung hat die menschlichen Arbeitsanteile sowohl in den kognitiven als auch manuellen Routineaufgaben verringert, während sie die Arbeitsanteile in den nicht-Routineaufgaben erhöht hat. Diese Veränderungen erklären außerdem einen großen Teil der Nachfrageverschiebungen zu immer mehr Hochqualifizierten (college graduates) im Arbeitsmarkt mit den bereits erwähnten Lohnund Verteilungseffekten.

Spitz-Oener (2006) greift den Ansatz von Autor et al. (2003) auf und untersucht, ob sich mit der Computerisierung auch die Qualifikationsanforderungen innerhalb der Berufe verändert haben. Hierzu 
kann sie auf ausführliche Daten aus Deutschland zurückgreifen, die die Arbeitsprozesse detailliert im Hinblick auf Aufgaben und Anstrengungen abbilden. Ihre Analyse setzt im Jahr 1979 an, was sie als Zeit vor der (M assen-)Computerisierung definiert, und beobachtet den Arbeitsmarkt über 20 Jahre hinweg. Die Komplexität hat in dieser Zeit am stärksten in Berufsfeldern zugenommen, in denen die Computerisierung rasch und umfangreich Einzug hielt. Dies hat auch die steigende Nachfrage nach hochqualifizierten Beschäftigten befördert. Auf Basis der gleichen Daten greift Spitz-Oener (2008) außerdem erneut die Frage der individuellen Lohnerträge aus der Computernutzung auf. Das Fazit von DiNardo und Pischke (1997) war, dass unbeobachtete Heterogenität und nicht der Computer die Lohnsteigerungen bei den Höherqualifizierten erklärte. Spitz-Oener (2008) kann auf Grundlage ihrer differenzierten Daten zeigen, dass die Computerisierung unter Berücksichtigung der Tätigkeitsprofile einen Einfluss hat. Im Gegensatz zu den traditionellen Arbeitsplatzmaterialien der Angestellten (white collar tools, d.h. Bleistifte u.ä., s.o.) hat der Computer zu einer Verstärkung der analytischen und interaktiven Aufgaben in den Angestelltenberufen geführt, also diese komplementär unterstützt und Kapazitäten, die vormals durch Routineaufgaben gebunden waren, freigesetzt. M itarbeiter mit Fähigkeiten, die zu Computern komplementär sind, haben daher höhere Löhne aufgrund ihrer erhöhten Grenzproduktivität, aber auch wegen der höheren Nachfrage nach ihren Fähigkeiten erhalten.

In einer ganzen Reihe von Studien wurden seitdem viele weitere Aspekte der Computerisierung auf die Änderungen der Beschäftigung und die Lohnungleichheit analysiert. Autor und Dorn (2013) stellen beispielsweise stagnierende oder sogar sinkende reale Einnahmen und Beschäftigungsanteile für die meisten geringqualifizierten Berufe fest. Zugleich finden sie Evidenz für eine gegenläufige Entwicklung bei den Dienstleistungsberufen. Hier zeigen sich nicht nur Beschäftigungszuwächse, sondern auch ein Lohnanstieg im Zeitraum von 1980 bis 2005. Bei differenzierter Betrachtung unter Berücksichtigung der Arbeitsinhalte bestätigen sich wiederum die unterschiedlichen Funktionen der Computerisierung (Substitution bzw. Ergänzung) als Ursache, die dadurch das Gesamtbild des polarisierenden Arbeitsmarkts befördern. Während Computer mehr und mehr die Routineaufgaben auch bei den geringqualifizierten Tätigkeiten übernommen haben, ist dies bei den Dienstleistungen nicht der Fall, da diese in der Regel Geschicklichkeit, Flexibilität, zwischenmenschliche Kommunikation und häufig auch physische Nähe erfordern. Dies impliziert, dass für die Dienstleistungen keine engen Substitute verfügbar sind. In der Folge finden sich steigende Löhne und Beschäftigung auch bei den geringqualifizierten Beschäftigten in diesem Bereich. Die stärkere Spezialisierung der menschlichen Arbeit auf Bereiche oder 
Tätigkeiten, die nicht durch die Computer ersetzt werden können, ist damit von grundlegender Bedeutung für die beobachteten steigenden Löhne und die steigende Beschäftigung auch im unteren Teil der Lohnverteilung. ${ }^{35}$

Dies kann zugleich als eine Bestätigung der von Baumol (1967) identifizierten Kostenkrankheit gesehen werden, auch wenn die Autoren darauf nicht eingehen. Nach Baumol ist nicht die spezifische Produktivitätsentwicklung in den Dienstleistungssektoren (bzw. den stagnierenden Sektoren) für die Lohn- und Gehaltsentwicklung entscheidend, sondern sie orientiert sich an der gesamtwirtschaftlichen Produktivitätsentwicklung. ${ }^{36}$ Entscheidend für das Ausmaß der Veränderungen ist das Ersatzpotenzial menschlicher Arbeit durch (digitale) M aschinen und die damit verbundenen Produktivitätsänderungen in den progressiven Sektoren. Dienstleistungen können daher ebenfalls bei Baumol progressive Sektoren sein, insofern sie routinierte bzw. automatisierbare Tätigkeiten enthalten.

Altonji et al. (2014) analysieren die Veränderungen in der Studienfachwahl aufgrund der Computerisierung, um die damit verbundenen Verschiebungen in der Struktur der Hochqualifizierten zu erklären. Ihre Ergebnisse machen deutlich, dass die Entwicklung der Ungleichheit in zwei Phasen unterschieden werden kann: während die Lohndifferentiale der Hochschulabsolventen zwischen 1993 und 2003 gestiegen sind und sich die Lohnungleichheit damit erhöht hat, ist sie in der folgenden Dekade zwischen 2003 und 2011 wieder geringer geworden. Die erste Phase war dabei durch einen steigenden Ertrag für abstrakte Aufgaben gekennzeichnet, die entsprechend als komplementär zu den Computern angesehen werden können. In der zweiten Periode sind die Erträge für diese Aufgaben aber rückläufig und es werden sogar leichte Zunahmen für Routineaufgaben festgestellt. Ein vergleichbar episodisches M uster finden auch Pikos und Thomsen (2016) bezüglich der Zunahme der Arbeitskomplexität und den damit verbundenen Lohnaufschlägen in einer Analyse für Deutschland für die Jahre 1986 bis 2012. Die Ergebnisse zeigen eine deutliche Zunahme der durchschnittlichen Zahl und Vielfalt der Aufgaben innerhalb eines Berufs. Während zusätzliche Aufgaben aber bis ungefähr zur Jahrtausendwende mit Lohnaufschlägen für die Beschäftigten verbunden waren, hat die zunehmende Normalität der Komplexität dazu geführt, dass trotz komplexerer Berufsbilder keine zusätzlichen Lohnaufschläge mehr erreicht werden.

\footnotetext{
${ }^{35}$ In ähnlicher Weise argumentieren u.a. auch Goos et al. (2014). Sie untersuchen Bestimmungsgründe für die Polarisierung der Arbeitsmärkte in 16 westeuropäischen Ländern. In ihrer Analyse gehen sie dabei auch auf die Bedeutung des Offshorings ein, das in die gleiche Richtung wie die Substitution von Routineaufgaben wirkt; d.h. Tätigkeiten werden zum Teil ersetzt, zum Teil aber auch ergänzt.

${ }^{36}$ Die gesamtwirtschaftliche Produktivität wächst dabei als gewichteter Durchschnitt der Wachstumsraten der progressiven und stagnierenden Sektoren. Stagnierende Sektoren reduzieren das gesamtwirtschaftliche Wachstum durch negative Wachstumsbeiträge.
} 
Mit verwandten Fragen beschäftigen sich auch Beaudry et al. (2014 und 2016). Sie gehen davon aus, dass sich der (US-)Arbeitsmarkt um die Jahrtausendwende gewandelt hat und dies zu den geringen Beschäftigungsquoten in der ersten Dekade des 21. Jahrhunderts führte. Während die Nachfrage nach hochqualifizierten Beschäftigten durch den Tech-Bust, der sich unter anderem im Platzen der DotCom-Blase (oder in Deutschland durch den Kollaps des Neuen M arktes) gezeigt hat, zurückgegangen ist, ist das Arbeitsangebot von College-Absolventen auch in den Folgejahren gestiegen. Die rückläufige Nachfrage hat dann zu einem Entwertungsprozess bzw. M ismatch der Qualifikationen am Arbeitsmarkt geführt. Höherqualifizierte haben Tätigkeiten übernommen, die ursprünglich von geringer qualifizierten Beschäftigten ausgeführt wurden, und haben hierfür im Durchschnitt entsprechende Lohneinbußen erfahren. Durch diesen Prozess hat zugleich eine Verdrängung „von oben“ eingesetzt, die geringer qualifizierte Beschäftigte die berufliche Leiter weiter hinabgedrückt oder gar aus dem Arbeitsmarkt geschoben hat. In einer Art Kaskadeneffekt haben sich so die Qualifikationen durch den Arbeitsmarkt bewegt. Nur die Höchstqualifizierten (Post-College-Absolventen) sind von dieser Entwicklung ausgenommen.

Hierzu gehen Beaudry et al. (2014) vor allem auf die Berufseinsteiger ein und können die Muster insbesondere für Collegeabsolventen seit der Jahrtausendwende zeigen. Beaudry et al. (2016) nehmen eine umfangreichere Analyse vor und kommen zu den Schlussfolgerungen, dass neben dem Rückgang der Nachfrage nach kognitiven Fähigkeiten auch der strukturelle Rückgang der Beschäftigung in Routineberufen und Fertigungsaufgaben sowie zyklische Muster durch die Finanzkrise eine Rolle für die Beschäftigungsverschiebungen spielen. Alle diese Aspekte spiegeln sich in den Substitutionspotenzialen der Computerisierung wider, die mehr Kapazitäten für andere menschliche Tätigkeiten ermöglicht hat.

Reinhold und Thomsen (2017) untersuchen, ob eine ähnliche Entwicklung auch in Deutschland beobachtet werden kann. Auf der Grundlage umfangreicher administrativer Daten für die Jahre 1975 bis 2010 berechnen sie eine Reihe von Arbeitsmarktindikatoren und untersuchen die Beschäftigungsmuster von Berufseinsteigern. Die empirischen Ergebnisse zeigen, dass sich der Anteil von hochqualifizierten Berufseinsteigern in höherbezahlten Tätigkeiten seit dem Jahr 2000 verringert hat. Deutlich wird überdies, dass bis zum Jahr 2010 die Einstiegsgehälter gesunken sind und sich das individuelle Lohnwachstum in den ersten fünf Berufsjahren verringert hat. Ein Grund dafür liegt in der Beschäftigung von Absolventen in Tätigkeiten, die früher von Personen mit geringeren Bildungsabschlüssen ausgeführt wurden. Diese Ergebnisse zeigen daher eine gewisse Ähnlichkeit zu den Entwicklungen des USamerikanischen Arbeitsmarkts, allerdings mit einem Unterschied der betroffenen Bildungsniveaus: während im US-amerikanischen Arbeitsmarkt die College-Absolventen betroffen sind, sind in Deutschland vor allem die Berufseinsteiger mit mittleren Qualifikationen beeinträchtigt. 


\section{Erwartete Entwicklungen und Implikationen für die Verteilung}

Die Ergebnisse zu den Wirkungen der Digitalisierung für den Arbeitsmarkt machen deutlich, dass diese bisher nicht folgenlos für die Verteilung der Löhne und Gehälter war. Die Arbeitsmarktentwicklung ist im betrachteten Zeitraum und insbesondere in der M ehrzahl der entwickelten Länder positiv verlaufen. Dennoch ist der Begriff der Digitalisierung nicht allein positiv konnotiert. Ergänzt werden muss, dass neben dem technischen Fortschritt durch die Digitalisierung in der gleichen Zeit auch die gestiegene Globalisierung (verbunden mit intensiverem Outsourcing und Offshoring), eine größere Arbeitsmarktflexibilisierung und Deregulierung (erkennbar in dem zurückgegangenen Einfluss von Gewerkschaften und Tarifverträgen) sowie neue Formen der Beschäftigung (die sogenannten Plattformwirtschaft, die über virtuelle Plattformen im Internet Nachfrage und Angebot von (Teil-)Aufgaben vermittelt) Einflüsse auf die Lohnverteilung gehabt haben (Eichhorst et al., 2016). ${ }^{37}$

Ungeachtet dieser begleitenden Einflüsse prognostizieren jüngere, viel beachtete Veröffentlichungen eine düstere Zukunft für die Beschäftigung durch Computerisierung und Digitalisierung. Nach Frey und Osborne (2017) arbeiten ca. 47\% der Beschäftigten der USA in Berufen, die in den nächsten zehn bis 20 Jahren potenziell automatisierbar sein sollten. Die zugrundeliegende Annahme der Ersetzbarkeit ganzer Berufe aufgrund überwiegender automatisierbarer Tätigkeitsanteile ist hierbei sehr kritisch zu sehen. Differenziertere Analysen auf der Grundlage von Tätigkeitsstrukturen von Bonin et al. (2015) und Arntz et al. (2016) sehen eher 9\% der Arbeitsplätze im gleichen Zeitraum als potenziell ersetzbar an. ${ }^{38}$ Dennoch lösen solche Ergebnisse intensive Diskussionen über die Gefahren der Digitalisierung aus. $^{39}$

\footnotetext{
${ }^{37}$ Die Plattformökonomie spielt bisher nur eine untergeordnete Rolle bezogen auf die Zahl der Beschäftigten. Sie illustriert aber den Übergang vom abhängigen zum selbstständigen Beschäftigungsverhältnis, wobei letzteres eher ein „unabhängiges“ Beschäftigungsverhältnis darstellt. Kritisch hinterfragt werden muss hierbei die Einordnung in die Sozialversicherungssysteme und der mögliche problematische Preiswettbewerb, der bei fehlender Berücksichtigung (nationaler) Lohnaufschläge (zur Finanzierung des Steuer- und Sozialsystems) entstehen kann.

${ }^{38}$ Eine grundsätzliche Schwierigkeit bei der empirischen Verwendung des Tätigkeit-basierten Ansatzes zur Untersuchung von Einflüssen der Digitalisierung liegt in der Verfügbarkeit geeigneter Daten. Die in der Literatur verwandten Daten über die Tätigkeitsstrukturen von Beschäftigten wurden in der Regel nicht spezifisch für die Untersuchung der Einflüsse von Computerisierung und Digitalisierung erhoben. Die Zuordnung der beruflichen Tätigkeiten im Hinblick auf ihre Automatisierbarkeit erfolgt entsprechend ex post. Diese zum Teil subjektive Einteilung hat dann auch bedeutende Einflüsse auf die geschätzten Ergebnisse, siehe hierzu Rohrbach-Schmidt und Thiemann (2013).

${ }^{39}$ Die Angst vor technischem Fortschritt ist kein neues Phänomen. So haben beispielsweise die Neuerungen in der Textilindustrie in der industriellen Revolution zu einem massiven Widerstand der Arbeiter (Ludditen) und schließlich einem gewaltsamen M aschinensturm im Jahr 1811 geführt. Der Luddismus ist vor allem im englischen Sprachraum eine bis heute historischer Inbegriff von Technologiefeindlichkeit. Aktuellere Beispiele sind die Erklärung von US-Präsident John F. Kennedy, dass die Erhaltung der Vollbeschäftigung in Zeiten der Automatisierung die größte innenpolitische Herausforderung der 1960er Jahre sei (Lenz 2018).
} 
Die verfügbaren empirischen Fakten zu den Wirkungen der Digitalisierung legen allerdings nahe, dass es (bisher) keinen Anlass für übertriebene Ängste gibt. Arntz et al. (2017) kritisieren hierbei auch die Beschränkung der Automatisierungsstudien (z.B. Frey und Osborne 2017) auf das arbeitsplatzsparende Potenzial, während die Beschäftigungswirkungen durch eine steigende Produktnachfrage oder den Produktnachfragemultiplikator außer Acht gelassen werden. Entsprechende empirische Ergebnisse für Europa (Gregory et al., 2016) zeigen eine positive Nettobilanz der Beschäftigung für die Jahre 1999 bis 2010 trotz negativer Wirkungen der technologischen Substitution. Auch gibt es ungeachtet der relativen Nachfrageveränderungen (bislang) kaum Belege einer zunehmenden Verbreitung sogenannter atypischer Beschäftigungen (u.a. Befristungen, Teilzeitarbeit, Leiharbeit oder sozialversicherungsfreie Werkvertragsbeschäftigung) durch die Digitalisierung. Sowohl die Befristungsquote als auch der Anteil der Zeitarbeit an der Gesamtbeschäftigung sind in Deutschland zuletzt nicht mehr angestiegen (Stettes 2016). Eine klare Korrelation der Nutzung von Zeitarbeit mit dem Digitalisierungsgrad der Unternehmen oder eine überproportionale Befristungsquote in stark digitalisierten Branchen lässt sich ebenfalls nicht bestätigen. Diese Ergebnisse können als Indiz für die Wandelbarkeit menschlicher Arbeit auf individueller und gesamtgesellschaftlicher Ebene gesehen werden. Diese Wandelbarkeit erlaubt es, sich immer wieder (schneller oder langsamer) an die technologischen Entwicklungen anzupassen (Lenz 2018).

Die drohenden Veränderungen sind wohl weniger disruptiv, sondern vollziehen sich - bisher zumindest - eher evolutorisch im Sinne eines anhaltenden Strukturwandels (Eichhorst und Rinne 2017). Auch die im historischen Vergleich niedrigen Produktivitätswerte sind ein Indiz dafür, dass der technologische Wandel nicht sprunghaft stattfindet (Lenz 2018). Dies ist eine wichtige Differenzierung, die bereits Ricardo (1821) als zentral erkannte. Seine in den ersten Abschnitten von Kapitel 31 der Principles gezeigten Nachteile für die Beschäftigten entstehen nämlich insbesondere bei einer plötzlichen und überraschenden Einführung einer produktiven M aschine. Hierzu schreibt er entsprechend:

"The statements which I have made will not, I hope, lead to the inference that machinery should not be encouraged. To elucidate the principle, I have been supposing, that improved machinery is suddenly discovered, and extensively used; but the truth is, that these discoveries are gradual, and rather operate in determining the employment of the capital which is saved and accumulated, than in diverting capital from its actual employment." (Ricardo 1821, Ch. 31.25)

Also nur bei einer entsprechend schnellen Einführung gravierender technischer Neuerungen verringert sich die Möglichkeit der Wiedereinstellung rationalisierter Arbeitskräfte in anderen Bereichen der Wirtschaft und damit die Kompensation für die verlorengegangene Beschäftigung. Ricardo (1821) liefert zugleich - am zeitgemäßen Beispiel der Nahrung - den Grund für die evolutorische Entwicklung:

"With every increase of capital and population, food will generally rise, on account of its being more difficult to produce. The consequence of a rise of food will be a rise of wages, and every rise of wages will have a tendency to determine the saved capital in a greater proportion than 
before to the employment of machinery. Machinery and labour are in constant competition, and the former can frequently not be employed until labour rises." (Ricardo 1821, Ch. 31.26)

Der Einsatz der Maschinen hängt damit von der menschlichen Nachfrage ab; oder - im Sinne Schumpeters ausgedrückt - von der Durchsetzbarkeit. Die menschliche Nachfrage entwickelt sich selbst evolutorisch und determiniert damit auch den Entwicklungspfad der Umsetzung der Automatisierung. ${ }^{40}$ Hierbei spielen auch Engpässe der zu automatisierenden Aufgaben eine Rolle. ${ }^{41}$ Die Durchsetzbarkeit neuer Technologien ist außerdem durch rechtliche, gesellschaftliche und ethische Barrieren beschränkt (Bonin et al. 2015). Es ist aber gleichzeitig feststellbar, dass die Verbreitung digitaler Technologien stetig zunimmt: die Betrachtung des Digitalisierungsgrads der Arbeitsmittel zeigt eine deutliche Zunahme innerhalb der letzten fünf Jahre (Arntz et al. 2018). Hieraus ergeben sich Handlungserfordernisse im Hinblick auf die Anpassung der Arbeit an die technologischen Veränderungen (z.B. durch Bildung und Weiterbildung).

Der durch die Computerisierung eingeleitete und sich beschleunigende Strukturwandel ändert die Anforderungen an die Beschäftigten, was sich in Änderungen der Erwerbsformen zeigt. Obgleich das „Normalarbeitsverhältnis" in Deutschland (Vollzeit, unbefristet) recht stabil rund 40 Prozent der Beschäftigung ausmacht, haben sich in den vergangenen rund 20 Jahren die Verhältnisse für die übrigen Beschäftigten und ihre Beschäftigungsformen verändert (Zahlen aus Eichhorst et al. 2016). So ist der Anteil der Inaktiven im gleichen Zeitraum von 26 auf 18 Prozent zurückgegangen und neue Beschäftigungsformen wie M inijobs (5 Prozent) oder Leiharbeit (2 Prozent) haben Einzug gehalten. Zugleich hat sich auf die Zahl der Befristungen und nebenberuflichen Selbständigkeiten erhöht. Hierdurch ergeben sich Herausforderungen für das Sozialversicherungssystem, die nicht nur die Frage der Lohnersatzleistungen, sondern vor allem auch das Rentensystem durch fehlende Vorsorge(-möglichkeiten) betreffen.

Eine Besonderheit der Computerisierung und Digitalisierung ist ergänzend zu berücksichtigen: die globale Reichweite. Wesentliche Gefahren, die aus der Digitalisierung für die Verteilung von Löhnen und Vermögen drohen, entstehen aus der zunehmend ungleichen Verteilung der Eigentumsverhältnisse. Durch die global agierenden Unternehmen, die für den technologischen Fortschritt maßgeblich

\footnotetext{
${ }^{40}$ Ein weiterer Aspekt ist die soziale Akzeptanz der Automatisierung. Ein gutes Beispiel dazu ist die Gastronomie: „Die bloße Tatsache, dass Tätigkeiten des Bedienungspersonals theoretisch automatisiert werden können, heißt nicht, dass Gäste dies auch honorieren. Nicht wenige mögen den menschlichen Service dem vollautomatisierten Restaurant vorziehen, auch wenn letzteres günstiger sein mag. " (Lenz 2018, S. 9)

${ }^{41}$ Frey und Osborne (2017) unterscheiden hierbei zwischen a) Wahrnehmungs- und Manipulationsaufgaben, b) Aufgaben der kreativen Intelligenz und c) Aufgaben der sozialen Intelligenz. Für a) entsteht der Engpass, da das Erreichen der Tiefe und Breite der menschlichen Wahrnehmung ist durch die Robotik bisher nicht möglich ist. b) ist beschränkt, da diese Tätigkeiten eine gewisse Kreativität voraussetzen und nur schwer zu spezifizieren sind, auch aufgrund von Veränderungen der gesellschaftlichen Wahrnehmung für Kreativität sowie der Diskrepanz zwischen den Kulturen. c) erfordert das Verständnis einer sozialen Vernunft.
} 
sind, besteht die Aussicht, dass die Wohlfahrtsgewinne der Digitalisierung und die Beschäftigungsrisiken eher ungleich verteilt werden (Arntz et al., 2017; Eichhorst und Rinne, 2017). Der bereits seit mehreren Dekaden zu beobachtende Bedeutungsrückgang des Produktionsfaktors Arbeit am Nationaleinkommen in den entwickelten Ländern (u.a. Deutschland, UK, USA, Frankreich, Japan) impliziert eine gestiegene Bedeutung des Kapitals. Die fortschreitende Digitalisierung verstärkt diese Entwicklungen, den Kapitaleignern kommt entsprechend immer größere Verantwortung bzw. M acht zu; insbesondere, wenn sie die Gewinne der Automatisierung privat vereinnahmen (können). ${ }^{42}$ Freeman wählte hierzu den zugespitzten Titel: „Who owns the robots, rules the world!“ (Freeman, 2015). Eng verbunden mit der großen Interaktion von Digitalisierung und Globalisierung werden diese Prozesse, die bisher vor allem die entwickelten Länder betrafen, zukünftig zunehmend weltweit von Bedeutung sein (u.a. Petersen, 2015). Hierdurch werden auch die Fragen regionaler Ungleichheit an Bedeutung gewinnen. Global betrachtet könnte es dann doch zu einer schnellen Abfolge von tiefgreifenden technischen Neuerungen kommen, bei der gemäß der Argumentation von Ricardo dann „die Kompensation sogar dauerhaft der Freisetzung hinterherhinken und starke soziale und politische Spannungen erzeugen“ (Kurz 2017, S. 787) kann.

Hieraus ergeben sich wichtige gesellschaftliche und politische Handlungsnotwendigkeiten (Eichhorst und Rinne, 2017). Ein besonderes Augenmerk muss auf die wachsende Dominanz der Kapitaleinkommen gelegt werden. Eine Fortsetzung des Anstiegs der relativen Bedeutung der Kapitaleinkommen führt unweigerlich zu einem Anstieg der Ungleichheit: dies gilt insbesondere dann, wenn die Besitzer des zukünftigen Kapitals die Gewinne der Automatisierung für sich vereinnahmen können ohne sich an den Kosten des Übergangs entsprechend zu beteiligen. Die zunehmende Substitution menschlicher Aufgaben durch M aschinen führt dabei zu einer langfristigen Verschiebung von Arbeits- zu Kapitaleinkommen. Dass die Digitalisierung hierbei eine Sonderrolle einnimmt, verdeutlichen Eichhorst und Rinne (2017, S. 16f.): „Die innovativsten Unternehmen, die in der digitalen Wirtschaft Märkte verändern oder erst schaffen und begründen, können im weltweiten M aßstab hohe Renditen erwirtschaften

\footnotetext{
${ }^{42}$ Arntz et al. (2018) analysieren hierzu ausführlich die Entwicklungen in Deutschland. Ihre Ergebnisse zeigen, dass die Digitalisierung zu einer Zunahme der technologischen Ungleichheit in der deutschen Betriebslandschaft führt. So setzen etwa 50 Prozent der deutschen Betriebe innovative Technologien ein. Diese Unternehmen haben durchschnittlich doppelt so viele Beschäftigte und erzielen höhere Umsätze. Zudem berichten sie deutliche Struktureffekte auf Berufs- und Branchenebene. Investitionen in neuartige Technologien führen zur Verdrängung von Arbeitsplätzen speziell in Berufen, die durch manuelle und kognitive Routineaufgaben sowie nicht-kognitive Routineaufgaben gekennzeichnet sind. Analytische und interaktive Berufe wurden durch die Investitionen befördert. Durch die positiven Produktnachfrageeffekte und der durch die Investitionen erreichten verbesserten Wettbewerbsfähigkeit der Unternehmen wuchs die Wirtschaft; die negativen Wirkungen wurden durch Substitutionseffekte überkompensiert. Für 2016-21 werden noch größerer Effekte der Technologieinvestitionen auf die Struktur der Beschäftigung erwartet. Darüber hinaus wuchs auch die Beschäftigungs- und Lohnungleichheit, wobei die Ungleichheit in den vergangenen fünf Jahren durch die betrieblichen Technologieinvestitionen begünstigt wurde. Hier hat eine signifikante Erhöhung der Beschäftigung und Löhne in Branchen und Berufen stattgefunden, die hoch entlohnt werden.
} 
- typischerweise zugunsten ihrer jeweiligen Gründer („the winner takes it all“).“ ${ }^{43}$ Diese weltweite Betrachtung bestätigt - wenn auch bei Ricardo eher als Warnung formuliert - die möglichen desaströsen Folgen lokaler Beschäftigung durch den Export von Maschinen:

"The employment of machinery could never be safely discouraged in a State, for if a capital is not allowed to get the greatest net revenue that the use of machinery will afford here, it will be carried abroad, and this must be a much more serious discouragement to the demand for labour, than the most extensive employment of machinery; for, while a capital is employed in this country, it must create a demand for some labour; machinery cannot be worked without the assistance of men, it cannot be made but with the contribution of their labour. By investing part of a capital in improved machinery, there will be a diminution in the progressive demand for labour; by exporting it to another country, the demand will be wholly annihilated." (Ricardo 1821, Ch. 31.29)

Um die resultierende Einkommensungleichheit zu reduzieren, kann das progressive Steuersystem als Korrektiv angepasst werden. Dies reicht aber nicht aus, um die sozialen Fragen zu lösen. Daher sollten die Sozialversicherungen stärker in den Veränderungsprozess einbezogen werden, da sich diese bisher maßgeblich über menschliche Arbeit finanzieren. Der sinkenden Bedeutung von Erlösen aus menschlicher Arbeit könnte durch eine stärkere Berücksichtigung der steigenden Bedeutung von Kapitaleinkommen begegnet werden. ${ }^{44} \mathrm{Um}$ die Beschäftigten auch direkt an den Veränderungen zu beteiligen, sollten auch Kapital- und Gewinnbeteiligungen der Beschäftigten in Betracht gezogen werden (Freeman, 2015). Unternehmen mit M itarbeiterbeteiligung haben sich dabei in der Vergangenheit als stabiler und robuster gegenüber konjunkturellen Schwankungen gezeigt; dies könnte insgesamt zu einer Verringerung der Ungleichheit beitragen. Die möglichen negativen Folgen (u.a. Trittbrettfahrerphänomen, hohe finanzielle Risiken für die Beschäftigten) müssten aber sorgsam abgewogen

\footnotetext{
${ }^{43}$ Schön beschrieben bereits von Ricardo: "The capitalist, I thought, was eventually benefited precisely in the same manner. He, indeed, who made the discovery of the machine, or who first usefully applied it, would enjoy an additional advantage, by making great profits for a time; but, in proportion as the machine came into general use, the price of the commodity produced, would, from the effects of competition, sink to its cost of production, when the capitalist would get the same money profits as before, and he would only participate in the general advantage, as a consumer, by being enabled, with the same money revenue, to command an additional quantity of comforts and enjoyments." (Ricardo 1821, Ch. 31.2)

${ }^{44}$ Dies entspricht auch den Empfehlungen von Baumol (siehe Hartwig und Krämer (2017)): Die relative Teuerung der Beschäftigung in den stagnierenden Sektoren impliziert nicht die Reduzierung dieser Sektoren, sondern erfordert genau im Gegenteil ihre - möglichst öffentlich geförderte - Ausweitung (insb. von Bildungs- und Gesundheitsdienstleistungen). Diese haben nämlich entscheidenden Beitrag zum Wirtschaftswachstum bzw. zur Entfaltung der Gesellschaft. Dennoch hat Baumols Kostenkrankheit eine wichtige soziale Implikation: die steigenden Preise für Dienstleistungen können nur von den Gesellschaftsmitgliedern adaptiert werden, deren Einkommen mit der oder stärker als die Produktivität wachsen. Wenn die unteren Einkommensklassen aber zu wenig von allgemeinen Einkommenszuwächsen profitieren, werden sie sich die Dienstleistungen nicht mehr leisten können. Die weniger Wohlhabenden werden immer mehr auf die durch den Staat bereit gestellten Dienstleistungen angewiesen, was zu Verteilungskonflikten zwischen den Steuerzahlern und Nichtsteuerzahlern führen kann. Inwiefern die erforderliche Umverteilung innerstaatlich gewährleistet werden kann, ist mit Blick auf die globale Dimension der Entwicklungen eine weitere, offene Frage.
} 
werden (Kruse, 2016). ${ }^{45}$ Ähnlich argumentieren auch Arntz et al. (2017). Sie sehen als kritischen Punkt der Entwicklung die Frage der Verteilung des Wachstums in der Gesellschaft. Hiervon hängen dann letztlich auch die (lokalen) Gesamtbeschäftigungswirkungen des digitalen Wandels ab: „Während steigende Lohneinkommen den lokalen Konsum stärken und damit neue Arbeitsplätze schaffen, könnten steigende Kapitaleinkommen weniger der lokalen Wirtschaft zu Gute kommen, wenn die Kapitaleigner im Ausland sitzen. Hier stellt sich die Frage, ob die geringeren Steuersätze auf Kapitaleinkommen im Vergleich zu Lohneinkommen eine Benachteiligung des Faktors Arbeit darstellen und ob durch eine Anpassung der relativen Steuerlasten die Beschäftigungswirkungen des digitalen Wandels positiv gestaltet werden können." (Arntz et al., 2017, S. 8f.).

Ein Verbot oder eine Sanktionierung technischen Fortschritts ist dabei - wir vor 200 Jahren bei Ricardo (1821) - allerdings keine Option. Schon er stellte fest:

"If, however, you were to reject the use of machinery, while all other countries encouraged it, you would be obliged to export your money, in exchange for foreign goods, till you sunk the natural prices of your goods to the prices of other countries. In making your exchanges with those countries, you might give a commodity which cost two days labour, here, for a commodity which cost one, abroad, and this disadvantageous exchange would be the consequence of your own act, for the commodity which you export, and which cost you two days labour, would have cost you only one if you had not rejected the use of machinery, the services of which your neighbours had more wisely appropriated to themselves." (Ricardo 1821, Ch. 31.30)

Die gegenwärtige Stimmung in Deutschland gibt hierzu aber auch keinen Anlass. Bisher sind die Einschätzungen der deutschen Bevölkerung zur Digitalisierung überwiegend positiv. Die Ergebnisse des ifo Bildungsbarometers 2017 (siehe Wößmann et al. 2017), einer repräsentativen Umfrage von erwachsenen Personen in Deutschland, zeigen, dass sich rund 54\% der Deutschen als Gewinner der Digitalisierung sehen; nur 16\% empfinden sich als Verlierer. Auch ist eine relative M ehrheit der M einung, dass es durch die Digitalisierung insgesamt mehr Gewinner als Verlierer gibt und dass sie eher nicht zu größerer Ungleichheit im deutschen Bildungssystem beitragen wird.

\section{Schlusswort}

Computer und Digitalisierung haben die Arbeitswelt über die vergangenen sechs Jahrzehnte verändert. Diese Änderungen waren dabei trotz des exponentiellen Fortschritts dieser Technologien weniger disruptiv, sondern eher evolutorischer Art. Arbeitsplätze und Arbeitsaufgaben haben sich entsprechend der technischen Möglichkeiten verändert, da Computer für eine Vielzahl von Tätigkeiten eingesetzt werden, die vorher von Menschen erbracht wurden. Die frei werdenden Kapazitäten konnten

\footnotetext{
${ }^{45}$ Andere Optionen, die diskutiert werden, sind Formen von Grundeinkommen oder auch eine Ausweitung öffentlicher, d.h. insbesondere staatlicher Beschäftigung. Diese sind aber in der Regel mit adversen Anreizen verbunden. Die wissenschaftlichen Ergebnisse zu vergleichbaren Subventionen sind überwiegend negativ.
} 
und können nun für andere, weniger leicht ersetzbare Aufgaben eingesetzt werden. Dies hat zu Verschiebungen in der Beschäftigungsstruktur geführt, die insbesondere die Enden der Lohnverteilung, also die geringen, aber auch die hohen Einkommen gestärkt hat. Die Erosion der mittleren Qualifikationen und Einkommen hat damit auch zu Ungleichheit geführt. Da zeitgleich mit der Computerisierung die Wirkungen anderer Prozesse die Arbeitsmärkte erfasst haben (Globalisierung, Deregulierung, neue Formen der Erwerbsarbeit), sind nicht alle Änderungen der Ungleichheit auf die Digitalisierung zurückzuführen. Sie hat sogar durch die ausgelösten Nachfrageprozesse bisher per Saldo zu mehr Beschäftigung geführt.

Dennoch hat der Anteil der Kapitaleinkommen im Vergleich zu den Arbeitseinkommen durch technische Substitution in allen entwickelten Ländern zugenommen. Der globale Charakter einer digitalisierten Welt wird diese Prozesse weiter verstärken. Dies impliziert, dass Kosten und Erträge ungleich anfallen: Kosten der Digitalisierung durch Beschäftigungsverluste treffen die lokalen oder nationalen Märkte, die Erträge fallen verstärkt bei den Eignern des Kapitals an und verteilen sich damit eher global. Der wirtschafts- und sozialpolitische Ausgleich dieser Ungleichverteilung durch verschiedene $\mathrm{M}$ aßnahmen ist für eine Reduzierung negativer Folgen der aus der Digitalisierung entstehenden Einkommens- und Vermögensungleichheit eine wichtige politische und gesellschaftliche Aufgabe.

\section{Literatur}

Acemoglu, Daron (2002), Technical Change, Inequality and the Labor M arket, Journal of Economic Literature, Vol. 40, Nr. 1, S. 7-72.

Acemoglu, Daron und David Autor (2011), Skills, Tasks and Technologies: Implications for Employment and Earnings, in: Ashenfelter, Orley und David Card (Hrsg.), Handbook of Labor Economics, Vol. 4b, Kapitel 12, S. 1043-1171, Elsevier Science, Amsterdam et al.

Acemoglu, Daron, David Autor, David Dorn, Gordon H. Hanson und Brendan Price (2014), Return of the Solow Paradox? IT, Productivity, and Employment in U.S. M anufacturing, NBER Working Paper No. 19837, Januar 2014.

Acemoglu, Daron und Fabrizio Zilibotti (2001), Productivity Differences, The Quarterly Journal of Economics, Vol. 116, Nr. 2, S. 563-606.

Aghion, Philippe (2002), Schumpeterian Growth Theory and the Dynamics of Income Inequality, Econometrica, Vol 70, Nr. 3, S. 855-882.

Altonji, Joseph G., Lisa B. Kahn und Jamin D. Speer (2014), Trends in Earnings Differentials across College Majors and the Changing Task Composition of Jobs, American Economic Review, Vol. 104, Nr. 5, S. 387-393.

Aristoteles (1880), Politik. Übersetzt von J.H. von Kirchmann, Philosophische Bibliothek Band 7, Verlag der Dürr'schen Buchhandlung, Leipzip. Verfügbar unter: http:// gutenberg.spiegel.de/ buch/politik-9246/1.

Arntz, M elanie, Terry Gregory und Ulrich Zierahn (2016), The Risk of Automation for Jobs in OECD Countries: A comparative analysis, OECD Social, Employment and M igration Working Papers, No. 189, OECD Paris.

Arntz, M elanie, Terry Gregory und Ulrich Zierahn (2017), Digitalisierung und die Zukunft der Arbeit, ifo Schnelldienst, Vol. 70, Nr. 7, S. 6-9. 
Arntz, M elanie, Terry Gregory und Ulrich Zierahn (2018), Digitalisierung und die Zukunft der Arbeit: Makroökonomische Auswirkungen auf Beschäftigung, Arbeitslosigkeit und Löhne von morgen, Abschlussbericht, Bundesministerium für Bildung und Forschung, Berlin.

Autor, David H. (2013), The "Task Approach" to Labor M arkets: an Overview, Journal for Labour M arket Research, Vol. 46, Nr. 3, S. 185-199.

Autor, David H. (2015), Why are There Still so Many Jobs? The History and Future of Workplace Automation, Journal of Economic Perspectives, Vol. 29, Nr. 3, S. 3-30.

Autor, David H. und David Dorn (2013), The Growth of Low-Skill Service Jobs and the Polarization of the US Labor Market, American Economic Review, Vol. 103, Nr. 5, S. 1553-1597.

Autor, David H., David Dorn, Gordon H. Hanson und Jae Song (2014), Trade Adjustment: Worker Level Evidence, The Quarterly Journal of Economics, Vol. 129, Nr. 4, S. 1799-1860.

Autor, David H., Frank Levy und Richard J. M urnane (2003), The Skill Content of Recent Technological Change: An Empirical Exploration, The Quarterly Journal of Economics, Vol. 118, Nr. 4, S. 12791334.

Autor, David H., Lawrence F. Katz und M elissa S. Kearney (2006), The Polarization of the US Labor M arket, American Economic Review, Vol. 96, Nr. 2, S. 189-194.

Autor, David H., Lawrence F. Katz und M elissa S. Kearney (2008), Trends in U.S. Wage Inequality: Revising the Revisionists, The Review of Economics and Statistics, Vol. 90, Nr. 2, S. 300-323.

Baldwin, Richard (2016), The Great Convergence - Information Technology and the New Globalization, The Belknap Press of the Harvard University Press, Cambridge(M A), London(UK).

Baumol, W. (1967). M acroeconomics of Unbalanced Growth: The Anatomy of Urban Crisis. The American Economic Review, 57(3), 415-426.

Baumol, William (2013). The Cost Disease: Why Computers Get Cheaper and Health Care Doesn't, Yale University Press.

Beaudry, Paul, David A. Green und Benjamin M. Sand (2014), The Declining Fortunes of the Young since 2000, American Economic Review, Vol. 104, Nr. 5, S. 381-386.

Beaudry, Paul, David A. Green und Benjamin M. Sand (2016), The Great Reversal in the Demand for Skill and Cognitive Tasks, Journal of Labor Economics, Vol. 34, Nr. S1, S. 199-247.

Becker, Gary (1993), Human Capital: A Theoretical and Empirical Analysis with Special Reference to Education, 3. Auflage, University of Chicago Press, Chicago.

Ben-Porath, Yoram (1967), The Production of Human Capital and the Life-Cycle of Earnings, Journal of Political Economy, Vol. 75, Nr. 4, S. 352-365.

Bonin, Holger, Terry Gregory und Ulrich Zierahn (2015), Übertragung der Studie von Frey/Osborne (2013) auf Deutschland, ZEW Kurzexpertise, N. 57, Mannheim.

Borghans, Lex und Bas ter Weel (2004), Are computer skills the new basic skills? The returns to computer, writing and math skills in Britain, Labour Economics, Vol. 11, Nr. 1, S. 85-98.

Bresnahan, T. F., und Trajtenberg, M . (1995), General Purpose Technologies: Engines of Growth? , Journal of Econometrics, 65(1), S. 83-108.

Brynjolfsson, Erik (1993), The productivity paradox of information technology, Communications of the ACM, Vol. 36, Nr. 12, S. 66-77.

Brynjolfsson, Erik und Andrew McAfee (2015), The Second Machine Age - Wie die nächste digitale Revolution unser aller Leben verändern wird, 2. Auflage, Börsen-M edien, Kulmbach.

Card, David und John E. DiNardo (2002), Skill-Biased Technological Change and Rising Wage Inequality: Some Problems and Puzzles, Journal of Labor Economics, Vol. 20, Nr. 4, S. 733-783.

David, Paul A. (1990), The Dynamo and the Computer: An Historical Perspective on the M odern Productivity Paradox, The American Economic Review, Vol. 80, Nr. 2, S. 355-361. 
Dewan, Sanjeen und Kenneth L. Kraemer (1998), International dimensions of the productivity paradox, Communications of the ACM , Vol. 41, Nr. 8, S. 56-62.

DiNardo, John und Jörn-Steffen Pischke (1997), The Returns to Computer Use Revisited: Have Pencils Changed the Wage Structure Too?, The Quarterly Journal of Economics, Vol. 112, Nr. 1, 291-303.

Dustmann, Christian, Bernd Fitzenberger, Uta Schönberg und Alexandra Spitz-Oener (2014), From sick man of Europe to economic superstar: Germany's resurgent economy, Journal of Economic Perspectives, Vol. 28, Nr. 1, s. 167-188.

Dustmann, Christian, Johannes Ludsteck und Uta Schönberg (2009), Revisiting the German wage structure, The Quarterly Journal of Economics, Vol. 124, Nr. 2, S. 843-881.

Eichhorst, Werner, Holger Hinte, Ulf Rinne und Verena Tobsch (2016), How Big is the Gig? Assessing the Preliminary Evidence on the Effects of Digitalization on the Labor Market, IZA Policy Paper No. 116, IZA Bonn.

Eichhorst, Werner und Ulf Rinne (2017), Der digitale Gestaltungsauftrag, ifo Schnelldienst, 70(7), S. 1618.

Fierheller, George (2014), Do not fold, spindle or mutilate: the "hole" story of punched cards, Steward Publishing, http://www.gfierheller.ca/wp-content/uploads/2014/02/Do-Not-Fold-Feb-7-2014web.pdf.

Fitzenberger, Bernd, Karsten Kohn und Qingwai Wang (2011), The erosion of union membership in Germany: determinants, densities, decompositions, Journal of Population Economics, Vol. 24, Nr. 1, S. 141-165.

Freeman, Richard (2015), Who owns the robots rules the world, IZA World of Labor 2015, 5 doi: 10.15185/izawol.5.

Frey, Carl Benedikt und Michael A. Osborne (2017), The future of employment: How susceptible are jobs to computerisation?, Technological Forecasting and Social Change, Volume 114, January, S. 254-280.

Gernandt, Johannes und Friedhelm Pfeiffer (2007), Rising Wage Inequality in Germany, Journal of Economics and Statistics, Vol. 227, Nr. 4, S. 358-380.

Gordon, Robert (2016), The Rise and Fall of American Growth - The U.S. Standard of Living since the Civil War, Princeton University Press.

Goos, Maarten und Alan Manning (2007), Lousy and Lovely Jobs: the Rising Polarization of Work in Britain, The Review of Economics and Statistics, Vol. 89, Nr. 1, S. 118-133.

Goos, M aarten, Alan M anning und Anna Salomons (2009), Job Polarization in Europe, American Economic Review, Vol. 99, Nr. 2, S. 58-63.

Goos, Maarten, Alan M anning und Anna Salomons (2014), Explaining Job Polarization: Routine-Biased Technological Change and Offshoring, American Economic Review, Vol. 104, Nr. 8, S. 2509-2526.

Gregory, Terry, Anna Salomons und Ulrich Zierahn (2016), Racing With or Against the M achine? Evidence from Europe, ZEW Discussion Paper No. 16-053, M annheim.

Hartwig, Jochen und Hagen Krämer (2017), 50 Jahre Baumol'sche Kostenkrankheit, Wirtschaftsdienst, 97 (11), S. 793-800.

Katz, Lawrence F. und Kevin M. M urphy (1992), Changes in relative wages, 1963-1987: supply and demand factors, The Quarterly Journal of Economics, Vol. 107, Nr. 1, S. 35-78.

Katz, Lawrence F. und David Autor (1999), Changes in the wage structure and earnings inequality, in: Ashenfelter, Orley und David Card (Hrsg.), Handbook of Labor Economics, Vol. 3, S. 1463-1555, Elsevier Science, Amsterdam et al.

Keynes, John Maynard (1936), The General Theory of Employment, Interest and Money, Palgrave McM illan, Basingstoke.

Kohn, Karsten (2006), Rising Wage Dispersion, After All! The German Wage Structure at the Turn of the

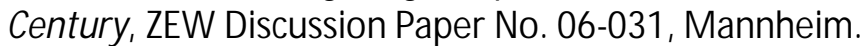


Krueger, Alan (1993), How Computers Have Changed the Wage Structure: Evidence from M icrodata, 1984-1989, The Quarterly Journal of Economics, Vol. 108, Nr. 1, 33-60.

Krugman, Paul (1994), Past and prospective causes of high-unemployment, Economic Review (Kansas), Vol. 79, S. 23.

Kruse, Douglas (2016), Does Employee Ownership Improve Performance?, IZA World of Labor 2016, 311, doi: 10.15185/izawol.311 1.

Kurz, Heinz D. (2017), Auf der Schwelle zur „Vierten Industriellen Revolution“, Wirtschaftsdienst, 97 (11), S. 785-792.

Lemieux, Thomas (2006), Postsecondary Education and Increasing Wage Inequality, American Economic Review, Vol. 96, Nr. 2, S. 195-199.

Lenz, Fulko (2018), Digitalisierung und Beschäftigung - Ein Ende ohne Arbeit oder Arbeit ohne Ende?, Argumente zu M arktwirtschaft und Politik, Nr. 141, Stiftung M arktwirtschaft, Berlin.

M arx, Karl (1968), Das Kapital, Karl M arx - Friedrich Engels - Werke, Band 23, Dietz Verlag, Berlin/DDR.

M ikfeld, Benjamin (2017), Digitale Transformation und die Arbeitswelt der Zukunft - Diskurse über den Wandel von Wirtschaft, Gesellschaft und Arbeit im digitalen Zeitalter, Diskussionspapier aus der Kommission "Arbeit der Zukunft", Hans Böckler Stiftung, M ai 2017.

M ilanovic, Branko (2016), Global Inequality - A New Approach for the Age of Globalization. The Belknap Press of the Harvard University Press, Cambridge(M A), London(UK).

Mincer, Jacob (1974), Schooling, Experience and Earnings, National Bureau of Economic Research, Cambridge.

M yck, Michal, Richard Ochmann und Salmai Qari (2011), Dynamics in transitory and permanent variation of wages in Germany, Economics Letters, Vol. 113, Nr. 2, S. 143-146.

Petersen, Thieß (2015), Globalisierung, Digitalisierung und Einkommensungleichheit, Bertelsmann Stiftung Policy Brief 2015/01, Gütersloh.

Pikos, Anna Katharina und Stephan L. Thomsen (2016), Rising W ork Complexity but Decreasing Returns, IZA Discussion Paper No. 9878, IZA Bonn.

Reinhold, Mario und Stephan L. Thomsen (2017), The changing situation of labor market entrants in Germany: A long-run analysis of wages and occupational patterns, Journal for Labour Market Research, S. 1-14, online first, doi:10.1007/s12651-017-0227-3.

Ricardo, David (1821), On the Principles of Political Economy and Taxation, 3. Auflage, London: John Murray.

Rohrbach-Schmidt, Daniela und M ichael Tiemann (2013), Changes in Workplace Tasks in Germany: Evaluating Skill and Task Measures, Journal for Labour Market Research, Vol. 46, Nr. 3, S. 215237.

Schumpeter, Joseph Alois (1997), Theorie der wirtschaftlichen Entwicklung, 9. Auflage, 1997 unveränderter Nachdruck der 4. Auflage von 1934, Duncker und Humblot, Berlin.

Smith, Adam (1776), An Inquiry into the Nature and Causes of the Wealth of Nations, Glasgow.

Solow, Robert (1957), Technical Change and the Aggregate Production Function, Review of Economics and Statistics, Vol. 39, Nr. 3, S. 312-320.

Solow, Robert (1987), "We'd better watch out", New York Times Book Review, July 12, 1987.

Spitz-Oener, Alexandra (2006), Technical Change, Job Tasks, and Rising Educational Demands: Looking outside the Wage Structure, Journal of Labor Economics, Vol. 24, Nr. 2, S. 235-270.

Spitz-Oener, Alexandra (2008), The Returns to Pencil Use Revisited, Industrial and Labor Relations Review, Vol. 61, Nr. 4, S. 502-517.

Stettes, Oliver (2016), Arbeitswelt der Zukunft - Wie die Digitalisierung den Arbeitsmarkt verändert, IW-Analysen Nr. 108, Institut der Deutschen Wirtschaft, Köln. 
Weitzman, Martin L. (1998), Recombinant Growth, The Quarterly Journal of Economics, Vol. 113, Nr. 2, S. 331-360.

Wößmann, Ludger, Philipp Lergetporer, Elisabeth Grewenig, Franziska Kugler und Katharina Werner (2017): Fürchten sich die Deutschen vor der Digitalisierung? Ergebnisse des ifo Bildungsbarometers 2017, ifo Schnelldienst, 17/2017, 70. Jahrgang, S. 3-24.

Wulff Pabilonia, Sabrina und Cindy Zoghi (2005), Returning to the Returns to Computer Use, American Economic Review, Vol. 95, Nr. 2, S. 314-317. 